\title{
Characterizing gene tree conflict in plastome-inferred phylogenies
}

\author{
Joseph F Walker ${ }^{\text {Corresp., Equal first author, } 1 \text {, Nathanael Walker-Hale }}{ }^{\text {Equal first author, } 2}{ }^{2}$, Oscar M Vargas ${ }^{3}$, Drew A Larson ${ }^{4}$, Gregory W \\ Stull ${ }^{\text {Corresp. } 5}$ \\ 1 Sainsbury Laboratory (SLCU), University of Cambridge, Cambridge, United Kingdom \\ 2 Department of Plant Sciences, University of Cambridge, Cambridge, Cambridgeshire, United Kingdom \\ 3 University of California, Santa Cruz, Santa Cruz, United States \\ 4 University of Michigan - Ann Arbor, Ann Arbor, Michigan, United States \\ 5 Department of Botany, Smithsonian Institution, Washington, United States \\ Corresponding Authors: Joseph F Walker, Gregory W Stull \\ Email address: jfw52@cam.ac.uk, gwstull@gmail.com
}

Evolutionary relationships among plants have been inferred primarily using chloroplast data. To date, no study has comprehensively examined the plastome for gene tree conflict. Using a broad sampling of angiosperm plastomes, we characterize gene tree conflict among plastid genes at various time scales and explore correlates to conflict (e.g., evolutionary rate, gene length, molecule type). We uncover notable gene tree conflict against a backdrop of largely uninformative genes. We find alignment length and tree length are strong predictors of concordance, and that nucleotides outperform amino acids. Of the most commonly used markers, matK, greatly outperforms $r b c L$; however, the rarely used gene rpoC2 is the top-performing gene in every analysis. We find that rpoC2 reconstructs angiosperm phylogeny as well as the entire concatenated set of proteincoding chloroplast genes. Our results suggest that longer genes are superior for phylogeny reconstruction. The alleviation of some conflict through the use of nucleotides suggests that stochastic and systematic error is likely the root of most of the observed conflict, but further research on biological conflict within plastome is warranted given documented cases of heteroplasmic recombination. We suggest that researchers should filter genes for topological concordance when performing downstream comparative analyses on phylogenetic data, even when using chloroplast genomes. 
5 Joseph F. Walker ${ }^{1,5^{*}}$, Nathanael Walker-Hale ${ }^{2 *}$, Oscar M. Vargas ${ }^{3}$, Drew A. Larson ${ }^{4}$ and Gregory

W. Stull 5,6

${ }_{1}^{1}$ Sainsbury Laboratory (SLCU), University of Cambridge, Cambridge, UK

10 2Department of Plant Sciences, University of Cambridge, Cambridge, Cambridgeshire, United

11 Kingdom

12 3University of California, Santa Cruz, Santa Cruz, United States

13 "University of Michigan - Ann Arbor, Ann Arbor, Michigan, United States

$14{ }^{5}$ Department of Botany, Smithsonian Institution, Washington, DC 20013, USA

${ }^{6}$ Authors for correspondence: J. F. Walker (jfw52@cam.ac.uk) and G. W. Stull

\section{ABSTRACT}

*Equal contribution

Evolutionary relationships among plants have been inferred primarily using chloroplast data. To date, no study has comprehensively examined the plastome for gene tree conflict. Using a broad sampling of angiosperm plastomes, we characterize gene tree conflict among plastid genes at various time scales and explore correlates to conflict (e.g., evolutionary rate, gene length, molecule type). We uncover notable gene tree conflict against a backdrop of largely uninformative genes. We find alignment length and tree length are strong predictors of concordance, and that nucleotides outperform amino acids. Of the most commonly used markers, matK, greatly outperforms $r b c L$; however, the rarely used gene rpoC2 is the top-performing gene in every analysis. We find that rpoC2 reconstructs angiosperm phylogeny as well as the entire concatenated set of proteincoding chloroplast genes. Our results suggest that longer genes are superior for phylogeny reconstruction. The alleviation of some conflict through the use of nucleotides suggests that stochastic and systematic error is likely the root of most of the observed 
34

35

5

conflict, but further research on biological conflict within plastome is warranted given documented cases of heteroplasmic recombination. We suggest that researchers should filter genes for topological concordance when performing downstream comparative analyses on phylogenetic data, even when using chloroplast genomes.

Key words: Angiosperms, chloroplast, gene tree conflict, rpoC2, phylogenomics, plastome, matK, rbcL.

\section{INTRODUCTION}

(4)

phylogenetics, largely due to the ease with which chloroplast genes can be sequenced, assembled, and analyzed (Palmer, 1985; Taberlet et al., 1991). The majority of broad-scale phylogenetic studies on plants have used chloroplast genes (e.g., Chase et al., 1993; Soltis et al., 2000, 2011), and the resulting phylogenies have been used for countless comparative studies examining ancestral states, historical biogeography, and other evolutionary patterns. While older studies relied mostly on targeted genes such as $r b c L$ and $m a t K$, recent advances in DNA sequencing have drastically increased the ease and affordability of whole-chloroplast genome (i.e., plastome) sequencing (Moore et al., 2006; Cronn et al., 2008, 2012; Stull et al., 2013; Uribe-Convers et al., 2014), increasing the number of studies employing plastome-scale data for phylogenetic and comparative analyses (e.g., Jansen et al., 2007; Moore et al., 2007, 2010; Ruhfel et al., 2014; Stull et al., 2015; Gitzendanner et al., 2018; Hong-Tao et al., 2019). Nonetheless, the utility of plastid genes, as well as the entire plastome, is ultimately determined by the extent to which they reflect 'true' evolutionary relationships (i.e., the 'species tree') of the lineages in question (Doyle, 1992).

Phylogenomic conflict (i.e., the presence of conflicting relationships among gene trees in a genomic dataset) is now recognized as a nearly ubiquitous feature of nuclear phylogenomic studies (Smith et al., 2015). Gene tree conflict is commonly attributed to biological causes such as incomplete lineage sorting, hybridization, and gene duplication and loss (Maddison, 1997;

3 Galtier and Daubin, 2008; Smith et al., 2015; Walker et al., 2017; Vargas et al., 2017). The genes 4 within the plastome, however, are generally thought to be free of such biological sources of 
65 conflict. This is because the plastome is typically uniparentally inherited (maternally in

66 angiosperms, and at times paternally in conifers: Mogensen, 1996) and undergoes a unique form

67 of recombination that is not expected to result in conflicting gene histories within the plastome

68 (Palmer, 1983; Bendich, 2004; Walker et al. 2015).

In angiosperms, nonmaternal inheritance of the chloroplast has been reported (e.g.,

Smith, 1989; McCauley et al., 2007), and several surveys of pollen in flowering plants

(Corriveau and Coleman, 1988; Zhang et al., 2003) have documented plastid DNA in up to 18\% of the species examined, indicating potential for biparental inheritance and heteroplasmy (i.e., the presence of two or more different plastomes in a single organism, cell, or organelle). Heteroplasmy, which has been documented in multiple angiosperm species (e.g., Johnson and Palmer, 1988; Lee et al., 1988; Hansen et al., 2007; Carbonell-Caballero et al., 2015), creates an opportunity for heteroplasmic recombination, which could result in gene tree conflict in plastome-inferred phylogenies. Unlike animal organelles, where there is little evidence for heteroplasmic recombination (White et al. 2008), and thus a strong justification for analyzing the mitochondrial genome as a single unit (Richards et al. 2018), heteroplasmic recombination (both intra- and interspecific) has been invoked by multiple studies to explain discordance in various clades (Huang et al., 2001; Marshall et al., 2001; Erixxon and Oxelman, 2008; Bouillé et al., 2011). However, only a few recent studies have documented clear evidence of this phenomenon (Sullivan et al., 2017; Sancho et al., 2018). Beyond heteroplasmy, sharing of genes between the chloroplast and nuclear or mitochondrial genomes remains another potential source of biological conflict (Martin et al., 1998; Martin, 2003; Stegemann et al., 2003; Rice et al., 2013; Straub et al., 2013; Smith, 2014), although transfer back to the plastome would be required to generate conflict and this has rarely been found (Smith, 2014). Even though biological conflict in the plastome generally seems rare, the full extent of intra-plastome conflict has yet to be evaluated. Quantifying its extent is of high importance given that most studies assume no conflict and treat the plastome as a single locus (Wolfe and Randle, 2004).

Aside from biological sources of conflict, there also remain significant potential sources of systematic and stochastic error that could contribute to conflict across the plastome (e.g., Burleigh and Mathews, 2007a,b). Chloroplast data are used at various time scales, and the accumulation of substitutions over long periods of evolutionary time increases the probability of encountering systematic error due to saturation (Rodríguez-Ezpeleta et al., 2007; Philippe et al., 
96 2011). Conflict has been demonstrated among different functional groups of genes (Liu et al., 97 2012), among different regions of the plastome (Davis et al., 2013; Walker et al., 2014), as well 98 as among individual genes (e.g., Shepherd et al., 2008, Foster et al. 2018; Gonçalves et al. 2019).

99 The rate of chloroplast evolution as a whole has been examined (and compared with the nuclear 100 and mitochondrial genomes; Wolfe, 1987), and rate variation within the chloroplast - especially across the three major regions of the genome, i.e., the large single-copy (LSC) region, the small

102 single-copy (SSC) region, and the inverted repeats (IRa, IRb) - has been explored to help 103 determine the markers useful for phylogenetic inference at different time scales (e.g., Graham and Olmstead, 2000; Shaw et al., 2005, 2007, 2014). However, no study has comprehensively examined gene tree conflict within the plastome to better characterize the extent and sources of conflict, and to identify the plastid genes most concordant with our current understanding of angiosperm phylogeny (e.g., Soltis et al., 2011; Wickett et al., 2014; Zeng et al., 2014; Gitzendanner et al., 2018; but see Logacheva et al., 2007 for a preliminary investigation of the concordance of individual plastid genes with angiosperm phylogeny).

Here we use phylogenomic tools to characterize the extent of conflict among plastid genes as a function of evolutionary rate, rate variation among species, sequence length, and data

112 type (i.e., nucleotides vs. amino acids) at varying time scales across angiosperms. Our results

113 show that plastid genes, when analyzed in combination, perform agree with many community 114 consensus relationships; however, across plastid genes there is notable gene tree conflict. The 115 number of conflicting genes at each node is often comparable to the number of concordant 116 genes; however, the majority of plastid genes are uninformative for most nodes when 117 considering support and thus unlikely to positively mislead studies. Information content (gene 118 length and molecule type, i.e., nucleotides vs amino acids) and evolutionary rate were positive 119 predictors of concordance, suggesting that most observed gene-tree conflict is a consequence of 120 spurious inferences from insufficient information. However, some nodes across angiosperm 121 phylogeny show numerous strongly supported conflicting genes; this rare event indicates an area 122 for future exploration. This may result from systematic error or could be the product of a 123 biological source of gene-tree conflict (e.g., heteroplasmic recombination, horizontal gene 124 transfer). We also document the performance of individual genes at recapitulating angiosperm 125 phylogeny, finding the seldom-used gene $r p o C 2$ to outperform commonly used genes (e.g., $r b c L$, 126 $m a t K$ ) in all cases, consistent with previous work highlighting the utility of this gene (Logacheva 
127 et al., 2007). Although as a whole the chloroplast infers a topology similar to currently

128 hypothesized angiosperm relationships, the conflict underlying it can influence branch lengths,

129 and therefore dating analyses, comparative methods, and other work that relies on branch lengths

130 could benefit from gene filtering procedures. Our results provide insight into the extent and

131 sources of intraplastome conflict.

132

133 MATERIALS AND METHODS

134 Data acquisition and sampling - Complete plastome coding data (both nucleotide and amino

135 acid) were downloaded from NCBI for 53 taxa: 51 angiosperm ingroups and two gymnosperm

136 outgroups (Ginkgo Biloba and Podocarpus lambertii; Table S1). Our sampling scheme was

137 designed to capture all major angiosperm lineages (e.g., Soltis et al., 2011) thus allowing

138 accurate inference of the angiosperm phylogeny, while also including denser sampling for nested

139 clades in Asterales. This allowed us to evaluate the extent of gene tree conflict at different

140 evolutionary levels/time scales, from species-level relationships in Diplostephium (Asteraceae)

141 to the ordinal-level relationships defining the backbone of angiosperm phylogeny.

142

143 Data preparation, alignment, and phylogenetic inference-All scripts and data used may be

144 found on GitHub (https://github.com/jfwalker/ChloroplastPhylogenomics). Orthology was

145 determined based upon the annotations of protein-coding genes on Genbank; this resulted in

146 near-complete gene occupancy apart from instances of gene loss or reported pseudogenization.

147 We did not use non-coding regions, as proper orthology at deep time scales can be difficult to

148 assess due to gene re-arrangements and inversions; this is in part why most deep phylogenetic

149 analyses of angiosperms using plastomes have excluded non-coding data (e.g., Moore et al.,

150 2010; Ruhfel et al., 2014).

151 The amino acid and nucleotide data were aligned using Fast Statistical Alignment (FSA

152 v1.15.9; Bradley et al., 2009) with the default settings for peptide and the setting "--noanchored"

153 for nucleotide. FSA has been shown to be one of the top-performing alignment programs

154 (Bradley et al., 2009; Redelings, 2014), and does not rely upon a guide tree for sequence

155 alignment, alleviating downstream bias (Bradley et al., 2009; Boyce et al., 2015; Chatzou et al.,

156 2018). A maximum likelihood (ML) tree was then inferred for each gene using RAxMLv.8.2.4

157 (Stamatakis, 2014), with the PROTGAMMAAUTO and GTR $+\mathrm{G}$ models of evolution used for 
158 the amino acid and nucleotide data, respectively. For each dataset, we conducted 200 rapid

159 bootstrap replicates. The alignments were also concatenated into supermatrices and partitioned

160 by gene using the phyx (v0.99) program pxcat (Brown et al., 2017).

161 The nucleotide and amino acid supermatrices were then each used to infer concatenated

162 'plastome' trees using the GTR $+\mathrm{G}$ and the PROTGAMMAAUTO models, respectively, as

163 implemented in RAxML. To complement the model inference performed by RAxML from the

164 AUTO feature, we also used IQ-TREE's (v1.5.4) (Nguyen et al., 2014) built-in model selection

165 process (Kalyaanamoorthy et al., 2017) on the partitioned data. We did not partition by codon

166 position because the lengths of most of the plastid genes, especially when divided into three

167 smaller partitions, would have been insufficient to inform an evolutionary model.

168 Recent work has advocated the use of multispecies coalescent methods when analyzing sets of 169 gene trees from the chloroplast (Gonçalves et al., 2019). As such we reanalyzed the nucleotide

170 data using the Maximum Quartets Support Species Tree method as implemented in Astral.v5.6.3

171 (Zhang et al., 2018) with default settings.

172

173

Reference phylogenies-For the conflict analyses, described below, we created several reference trees against which the gene trees were mapped. Primarily, we used a topology based on Soltis et al. (2011) and where possible that of Wickett et al. (2014), and - for species-level relationships within Asteraceae - Vargas et al. (2017). We refer to this as our 'accepted tree', or AT (Fig 1.). We also created a reference tree based on the recent plastome phylogeny of Gitzendanner et al. (2018), which we refer to as the 'Gitzendanner tree', or GT. The GT is highly concordant with the AT, but several deep (and commonly contentious) nodes differ, e.g., the placement of Buxales and ordinal relationships in lamiids (here, we only sampled Lamiales, Gentianales, and

182 Solanales). In the latter case, all three possible combinations of lamiids taxa were assessed

183 (discussed further below in Assessment of Supported Conflict). Although it is difficult to 184 determine which of these trees better represents species relationships of angiosperms, they both serve as useful frameworks for revealing conflict among genes. Here, we primarily focus on analyses using the AT, but conflict analyses using the GT were also conducted to ensure that our overall results were consistent across reference trees. 
189 Analysis of conflict -All gene trees were rooted on the outgroups using the phyx program pxrr 190 (Brown et al., 2017), in a ranked fashion (“-r") in the order Podocarpus lambertii then Ginkgo

191 biloba; this was performed to account for any missing genes in either of the taxa. In all cases the 192 trees were rooted with at least one gymnosperm. Gene tree conflict in the data was identified 193 using the bipartition method as implemented in phyparts v.0.0.1 (Smith et al. 2015), with the 194 gene trees from each data set (amino acid and nucleotide) mapped against the AT and GT 195 references trees. The concordance analyses were performed using both a support cutoff (at $\geq 70 \%$ 196 bootstrap support, i.e., moderate support as suggested by Hillis and Bull, 1993) and no support 197 cutoff. When the support cutoff is used, any gene tree node with less than $70 \%$ bootstrap support 198 is regarded as uninformative for the reference node in question (i.e., it is uncertain whether the gene tree node is in conflict or concordance with the reference node); when no support cutoff is used, the gene tree node is evaluated as conflicting or concordant regardless of the support value. However, in both cases, a gene is considered uninformative if a taxon relevant to a particular node/relationship is missing from the gene dataset. For example, consider the reference tree $((\mathrm{A}, \mathrm{B}), \mathrm{C})$; if a gene tree is missing taxon $\mathrm{B}$, but contains the relationship $(\mathrm{A}, \mathrm{C})$, then the gene tree is concordant with the node $((\mathrm{A}, \mathrm{B}), \mathrm{C})$ but uninformative regarding the node $(\mathrm{A}, \mathrm{B})$. See Smith et al. (2015) for an in-depth explanation of this method. To examine patterns of concordance and conflict among all gene trees, we calculated the pairwise Robison-Foulds distance (Robinson and Foulds, 1981) (RF) between each gene tree and the AT, concatenated topology, and ASTRAL tree (see below). We created a network of trees based on RF distance using the R package igraph v1.2.4.1 in R v3.6.1 (Csardi and Nepusz, 2006; R Core Team, 2018), using trees as nodes, inverse RF distance as edge weights, and the Fruchterman-Reingold algorithm to lay out the graph (Fruchterman and Reingold, 1991).

213 intervals of the evolutionary history of angiosperms. For the latter case, the $\sim 150$ Ma of crown

214 angiosperm evolution were divided into five time-intervals (30 Ma each): 150 to 121 mya, 120 to 21591 mya, 90 to 61 mya, 60 to 31 mya, and 30 to 0 mya. The nodes across the tree were binned into 216 the time intervals based on their inferred ages (ages > 20 Ma from Magallón et al., 2015; ages < $21720 \mathrm{Ma}$ from Vargas et al., 2017 and Roquet et al., 2009). At each time interval, the proportion of 218 concordant nodes for each gene was calculated (for all the nodes falling within that time 219 interval). This allowed us to assess the level(s) of divergence at which each gene is most 
220 informative. We also determined concordance levels of the 'plastome' trees (against the

221 reference) within each of these time intervals.

222

223

Assessments of Individual Plastid Genes - Using the phyx program pxlstr (Brown et al., 2017),

224

225

226

227

228

229

230

231

232

233

234

235

236

237

238

239

240

241

242

243

244

245

246

247

248

249

we calculated summary statistics for each gene alignment and corresponding gene tree: number of included species (as gene alignments could be missing a species from pseudogenization or gene loss), alignment length, tree length (a measure of gene evolutionary rate), and root-to-tip variance (a measure of rate variation across the phylogeny). Alignment length and tree length represent complementary measures of a gene's information content. All else being equal, longer genes should harbor more phylogenetic signal. Tree length serves as a model-informed metric because all sites, and not just those which are variable or parsimony informative, are used in estimation of model distances. Because our study uses maximum likelihood methods, we chose this model-informed metric as opposed to aforementioned metrics. Levels of concordance of each gene tree with the reference trees were then assessed by tabulating the number of nodes concordant between the gene tree and the given reference tree (e.g., the AT). The number of concordant nodes (in Fig. 2 treated as a proportion of total nodes available to support and in Fig. 3 based on total nodes) was used as a measure of a gene's ability to accurately reconstruct the angiosperm phylogeny.

Predictors of Concordance-We examined the statistical relationships between gene tree concordance (using concordance data from the AT-based conflict analysis) and alignment length, tree length, and root-to-tip variance. We considered each node as a trial and analyzed the concordance (success) or conflict (failure) in aggregate, such that each gene was a single observation with $x$ successes in $n$ trials. Because of variable sampling and loss of some genes, $n$ was variable between observations. We analyzed the relationship between probability of concordance and alignment length, tree length, and root-to-tip variance using logistic regression of aggregate binomial trials with the function $\operatorname{glm}()$ in R v3.4.4 (R Core Team, 2018). Binomial models were generally characterized by high residual deviance, and we thus allowed for overdispersion by fitting quasibinomial logistic regressions (using 'family = quasibinomial()' in $\mathrm{R}$ v3.4.4). We fitted models for both amino acid and nucleotide data, both not considering and 
250

251

252

253

254

255

256

257

258

259

260

261

262

263

264

265

266

267

268

269

270

271

272

273

274

275

276

277

278

279

280

considering support, and for both alignment methods. We also fitted models for amino acid data inferred only under WAG.

We modeled gene performance as a function of length, tree length, and root-to-tip variation, and as a function of each predictor individually. Because it is possible that apparent relationships between alignment length and concordance may reflect signal from gene information content per alignment site, we also modelled gene performance as a function of length and tree length (as a proxy of gene information content and rate, respectively, noted above), to assess the relationship between alignment length and gene performance after controlling for variation associated with rate.

Investigation of model fits on full datasets by studentized residuals, leverage and Cook's distance values revealed that several observations were highly influential. Therefore, we also conducted analyses on reduced datasets to investigate the influence of these observations. In amino acid datasets, we excluded $r p l 22$ and $r p l 32$, which were probably influential due to their high tree length values, and $y c f 1$ and $y c f 2$, which were probably influential based on their long alignments. In nucleotide datasets, we excluded $c l p P$ which had poor performance relative to tree and alignment length, rps 15, which had relatively long tree length, and $y c f 1$ and $y c f 2$ for the same reasons as previously. Combined analyses of alignment length and tree length were not subject to influence driven by high root-to-tip variance values, and hence reduced datasets had fewer genes removed. In this case, we excluded only $y c f 1$ and $y c f 2$. Regression results were summarized in tables using the R package Stargazer (Hlavac, 2018).

Saturation Analyses-We also performed saturation analyses on all the chloroplast genes to determine if they were capable of inferring deep divergences (Phillipe et al, 1994). In this analysis the pairwise distances of the sequences are compared to the model-corrected distances as a means of identifying if the real genetic distances may be properly estimated. The saturation analysis was performed on each gene and each codon position and thus the data were realigned by codons for this analysis. The amino acid alignments were used to guide the codon alignments using the program pxaa2cdn, from the phyx package. Four of the genes did not match their corresponding amino acid sequence in its entirety and these were left out of this analysis, since the codons could not be properly aligned. This discrepancy is likely due to errors in the GenBank submission (the amino acid and nucleotide data do not perfectly correspond). Saturation was 
281 assessed by determining the observed number of differences between sequences compared to the

282 inferred number of substitutions. This analysis was performed using the "dist.dna" and

283 “dist.corrected" functions in the R package "ape" (Paradis et al. 2018), with the F84 model of

284 evolution (Felsenstein and Churchill, 1996) used for the correction.

285

286 Comparison of genomic regions-We assessed the utility of the three major plastome regions-

287 the Large Single Copy (LSC) region, Small Single Copy (SSC), and the Inverted Repeat (IR)

288 region - for reconstructing angiosperm phylogeny in two ways. First, we constructed ML

289 phylogenies by concatenating all genes found in each region (as described above for each of the

290 plastome trees inferred using all genes). We then calculated the number of concordant nodes

291 with the AT for the resulting genomic regions trees. Second, using the concordance levels of

292 each individual gene (described above), we created a plastome diagram (with genes arranged

293 according to their genomic position) showing the concordance levels of each gene at the five

294 different time scales discussed above (Fig. S1); this permits a qualitative visual assessment of the

295 general concordance levels of each genomic region at each time slice.

296

297 Assessment of supported conflict-We compared four contentious regions of the AT to a series

298 of alternative relationships. The tested alternative relationships were present in either the GT

299 (i.e., the plastome phylogeny of Gitzendanner et al., 2018), our plastome phylogenies (with $\geq 70$

300 BS for the node in question; we refer to this tree below as the 'plastome tree', or PT), and/or

301 three or more of the gene trees from our nucleotide dataset (with $\geq 70 \mathrm{BS}$ for the node in

302 question). These alternative relationships pertain to: (1) the placements of Buxus and

303 Trochodendron; (2) relationships among Acnistus (Solanales), Coffea (Gentianales), and Olea

304 (Lamiales); (3) the placements of Jacobaea and Artemisia in Asteraceae; and (4) the placements

305 of Galinsoga, Ageratina, and Parthenium in Asteraceae. To test these alternative relationships,

306 we used a modified version of the Maxmimum Gene Wise Edge (MGWE) method (Walker et

307 al., 2018), where instead of a defined "TREE SET", we used a constraint tree for each alternative

308 hypothesis. The likelihood for every gene was calculated across each constraint, thereby forcing

309 the relationship in question to be the same. Similar to MGWE, this allowed for conflict across

310 the rest of the topology; however, this provides a greater amount of tree space to be explored 
311 (similar to Smith et al., 2018a). This method creates likelihood scores solely based on each

312 alternative relationship, making it robust to gene tree conflict outside of the node in question.

313 The modification of the MGWE method has been implemented in the EdgeTest.py program of

314 the package PHylogenetic Analysis Into Lineages (https://github.com/jfwalker/PHAIL).

315

316 Assessment of influence of alignment method-Because initial homology statements at the

317 alignment stage can strongly influence the outcome of phylogenetic inference (Morrison, 2009;

318 Morrison et al., 2015), we repeated the conflict analysis using the same parameters as above with

319 two alternate sets of alignments. In one case we re-inferred using MAFFTv2.271 (Katoh and

320 Standley, 2013) with the setting “_ auto — max_iterate 1000”. In the second case we cleaned the

321 original FSA gene alignments using pxclsq (Brown et al. 2017) for a minimum of $30 \%$ column

322 occupancy in order to remove gap-heavy and ambiguously aligned sites, and RAxMLv8 was

323 used.

324

325

Assessment of influence of model - To test if the inferred model of evolution influenced the

326

inference, we re-ran the tree inference using the same methods above on the uncleaned FSA

327

amino acid datasets using only the WAG model of evolution (Wheelan and Goldman, 2001). The

328

WAG model was not found to be the optimal amino acid model for any gene in the analysis, thus

329

this allowed us to assess the influence of assigning the same sub-optimal model across all

330 partitions.

331

332

\section{RESULTS}

333

334

Influence of alignment and model - The use of MAFFT as opposed to FSA as an alignment tool did not produce a qualitatively different result (Tables S2-7). Furthermore, our results were

336 largely not qualitatively influenced by using an alignment that had been cleaned for 30\%

337 minimum occupancy (Tables S2-6 and Tables S8-9). Quasibinomial regression results for amino

338 acids aligned with FSA and cleaned for 30\% minimum occupancy were not significant for all

339 predictors, but this model had an abnormally high estimated dispersion parameter (almost 17

340 times greater than the next largest estimate), suggesting that $\mathrm{p}$-values inferred under this model

341 were unreliable (Tables S2-5). When running a sub-optimal model of evolution for all genes (the 
342 WAG model) we recapitulated the results of our model-tested analysis (Tables S2-5 and Table

343 S10). The differences among all these analyses were occasionally off by one in terms of number

344 of concordant nodes; however, no results qualitatively changed: ranking of genes in terms of

345 concordance, inference from regression models and detection of strongly-supported conflict were

346 largely unchanged. Therefore, for the remainder of the results and discussion we reference the

347 uncleaned FSA alignment (Tables S11-12).

348

Patterns of conflicting chloroplast signal-The sampling for this experiment allowed us to examine conflict at multiple timescales (the values for each analysis may be found in Tables S612), with roughly 10 divergences occurring in each $30 \mathrm{Ma}$ bin. Our 'plastome' trees, inferred from the concatenated gene sets (nucleotide and amino acid), were highly concordant with the AT (Fig. 1 and Fig. S2-3). Without considering support, the gene trees showed high levels of conflict across the different analyses. This pattern is similar to that seen in nuclear phylogenomic datasets (Salichos and Rokas, 2013; Smith et al., 2015; Shen et al., 2016). When considering support, the majority of the plastid genes were uninformative for practically all nodes in the phylogeny (Fig. 1; Tables S11-12); i.e., they had bootstrap support below 70\% (moderate support) for that particular relationship (whether in conflict or concordance).

There was no obvious relationship between the amount of gene tree conflict and evolutionary scale (i.e., conflict was relatively evenly distributed across shallow and deeper nodes/time scales; Figs. 1, 2). Although the greatest degree of gene tree concordance with the AT appeared in the nodes with inferred ages between 90-61 Ma (ages based on Magallón et al., 2015), these nodes typically still contained at least 50\% uninformative gene trees (Fig. 1). Genome location also showed weak correspondence to conflict/concordance (Fig. S1; Table S13); discussed further below. Rather than timescale and genome location, data type (nucleotide vs amino acid) had a much greater impact on the prevalence of conflict (Figs. 2, 3), with the amino acid dataset generally showing higher levels of gene tree conflict. When factoring in support (BS $\geq 70 \%$ cutoff), the amino acid data set showed even less concordance with the AT (as more genes were considered uninformative due to low BS support). The integration of

370 support also decreased concordance of the nucleotide data set with the AT, but proportionally

371 less. See Fig. S2-3 for conflict analyses showing the amino acid and nucleotide gene trees

372 mapped onto the AT (the nucleotide results from Fig. S2 are also shown in Fig. 1). 
To identify how many different models of amino acid evolution underlie the genes in the

374

375

376

377

378

379

380

381

382

383

384

385

386

387

388

389

390

391

392

393

394

395

396

397

398

399

400

401

402

403 plastome, we tested each gene against the candidate set of amino acid models in IQ-TREE and RAxML. We found that a wide range of evolutionary models best fit the individual genesrather than just a single model that best fits every gene (Table S12). Many of the models were not designed specifically for plastome data, and cpRev, which was designed for plastome data, was only the best fit for 19 of the 79 genes based upon the IQ-TREE model test.

To examine relationships between gene characteristics and levels of concordance/conflict, we calculated the following statistics for each gene: alignment length (a measure of gene information content), tree length (a measure of evolutionary rate), and root-totip variance (a measure of variation in evolutionary rate across the tree), all of which can influence properties of phylogenetic inference (Shen et al. 2016). This information is presented for each gene across both data types in the supplementary information (Tables S11-12). We used logistic multiple regression to test relationships between concordance and gene characteristics (Tables S14-21). We found that alignment length had a significant positive multiplicative relationship with odds of concordance across both datasets (Fig. 2, Tables S14-17). Tree length and root-to-tip variance had significant positive and negative multiplicative relationships, respectively, in both datasets (Tables S14-15). Notably, excluding highly influential observations did not affect the direction or significance of most relationships (Tables S16-17), indicating that the results were not due to the influence of these data points. However, this did increase the estimated impact of alignment length on log odds by a factor of 10 , probably due to the high leverage exerted by the very long alignments of $y c f 1$ and $y c f 2$. In models including only alignment length and tree length, both predictors had significant positive multiplicative relationships with odds of concordance even when the other was included in the model (Fig. 2, Tables S18-21).

We examined patterns of conflict among individual genes by calculating the pairwise RF distance and producing a network graph (Fig. S4). Most genes had large pairwise RF distances to all other genes. We observed a general cluster of genes that were less distant from one another and from the AT and inferred species trees, including rpoC2 and $n d h F$, that appeared to correlate with alignment length (Fig. S1). In general, the tree set is characterized by high levels of discordance among gene trees as well as between gene trees and the AT and inferred species trees. 
405 Genomic patterns of concordance/conflict-In terms of number of nodes concordant with the

406 AT, the LSC and SSC regions were roughly comparable, with both outperforming the IR

407 regardless of whether BS support was considered (Table S13). This pattern held across time

408 periods, with the LSC and SSC regions having more concordant nodes than the IR at every time

409 slice. The tree lengths of the LSC and SSC regions (1.82 and 2.05, respectively) were also

410 considerably larger than that of the IR (0.98). The concatenated gene alignment lengths of the

411 LSC, SSC, and IR were 73,422 bp, 10,395 bp, and 19,314 bp, respectively. The genome

412 diagram of concordance (Fig. S1) does not show any striking patterns among the different

413 genomic regions; however, it is notable that the majority of the LSC region is discordant (or

414 uninformative) except for a few highly informative genes (namely, rpoC2 and matK).

415

416 Performance of individual plastid genes-Across all analyses, rpoC2 showed the highest levels

417 of concordance with the AT (Fig. 2, Tables S11-12); in general, when support was not

418 considered, it performed at least as well as the 79 concatenated genes in reconstructing the AT

419 (Fig. 3). The commonly used genes $n d h F$ and matK generally scored among the best-performing 420 plastid genes (in terms of number of concordant nodes), while $r b c L$, the other most commonly

421 used gene, performing relatively poorly (Fig. 2). The matK alignment is $\sim 250$ bp longer than the

$422 r b c L$ alignment; the best performing gene, $r p o C 2$ (alignment length 4,660 bp), is one of the

423 longest plastid genes. However, the notably long region $y c f 1$ - the unaligned sequence of which

424 is 5,400 bp (Dong et al., 2015) — did not perform as well as rpoC2. In this study, the alignment

425 length of $y c f 1$ was $21,696 \mathrm{bp}$ (vs. 4,660 bp for rpoC2). In several cases it performed toward the

426 top; however, it was never the top-performing gene in terms of number of concordant nodes

427 (Tables S11-12). Despite the high levels of observed conflict overall, we found that every node

428 of the AT was supported by at least one gene. Thus, to varying degrees, all relationships of the

429 AT are found within the plastome gene tree set.

430

431 Saturation analyses-None of the genes analyzed showed significant signatures of saturation

432 (Fig. S5). The uncorrected genetic distances and the corrected genetic distances appeared to be

433 roughly the same, and this trend was true for the first, second, and third codon position. Four

434 genes ( $n d h D$, psbL, rpl16, and $r p l 2)$ were unable to be properly analyzed for saturation as the 
435 nucleotide data uploaded to GenBank were missing the codons for 2 to 3 amino acids in some 436 taxa resulting in an improper codon alignment.

437

438 Analysis of well-supported conflict-We found that, out of the four major contentious 439 relationships in the AT, the edge-based analysis only supports one (Table 1) compared to the 440 alternative hypotheses examined (i.e., the GT, the plastome trees, and the gene trees). In the case 441 of Buxus (node 1 in Fig. 1), this analysis supports Buxus as sister to Trochodendraceae (Table 1), 442 which is the topology found in the GT and four gene trees: rbcL, petL, rps2, rps 14 (Table 2). In 443 the case of lamiids relationships (node 2), the data support Olea (Lamiales) sister to Acnistus 444 (Solanales) + Coffea (Gentianales), which is the relationship present in the GT and 15 gene trees 445 (Tables 1 and 2). For node 3 (the placements of Jacobaea and Artemisa), the data support the AT 446 topology, yet no individual gene trees provided $\geq 70$ BS support for this relationship; however, 447 rpoC2 matched the GT topology with strong support (Tables 1 and 2). Lastly, for node 4 (the 448 placement of Galinsoga), the data collectively support Parthenium argentatum sister to 449 Galinsoga (our plastome tree, PT), with two gene trees (petA, psaJ) providing strong support for 450 this topology; however, 16 genes (Table 2) supported a dominant alternative (Ageratina sister to 451 Galinsoga quadriradiata).

452

\section{DISCUSSION}

454 Following the typical assumptions of chloroplast inheritance, we would expect all genes in the 455 plastomes to share the same evolutionary history. We would also expect all plastid genes to show 456 similar patterns of conflict when compared to non-plastid inferred phylogenies. Furthermore, the 457 amino acid and nucleotide plastid data we used should show the same conflicting/concordant 458 relationships against given reference trees (e.g., the AT or GT). Our results, however, discussed 459 below, frequently conflict with these common assumptions about chloroplast inheritance and 460 evolutionary history.

461

462 Conflicting topologies inferred from the chloroplast genome - In general, the 'plastome' 463 topologies inferred from nucleotide and amino acid alignments showed high levels of 464 concordance with the AT (Fig. 1; Tables S11-12). While the genes within the chloroplast 465 genome are largely uninformative for most nodes of the phylogeny, a number of genes exhibited 
466 well-supported conflict (Fig. 1). In general, there appears to be no relationship between

467 evolutionary scale and amount of gene tree conflict (i.e., conflict generally does not appear to

468 correlate with divergence time in angiosperms). Instead, the extent of conflict/concordance had a

469 stronger relationship with the molecule type analyzed. The amino-acid dataset showed the

470 highest levels of gene tree conflict (Fig. 3, Table S12), and the nucleotide dataset had about half

471 the amount of gene tree conflict found in the amino acid data (Fig. 3, Table S11). Support from

472 edge-based analyses (a topology test that is robust to gene tree conflict at nodes other than the

473 one under consideration) for the GT over the AT suggest that at least some signal for conflicting

474 relationships is derived from underlying gene tree conflict when resolving species relationships

475 using all genes. Positive relationships between alignment length, tree length and concordance

476 also suggest that stochastic error is at play, because shorter or slower-evolving genes harbor less

477 signal to accurately infer relationships. Pairwise comparisons between chloroplast genes

478 indicated that they did not appear to share the same evolutionary history (Fig. S4). Even longer

479 genes were relatively discordant with one another (for example rpoC2 and $y c f 1$ had an RF

480 distance of 27). Against a backdrop of largely uninformative genes, long, influential sequences

481 might drive the results of concatenated analyses (Shen et al., 2016). Indeed, rpoC2 and $n d h F$ had

482 small distances to the AT, concatenated topology, and to one another. Because the AT is a

483 synthetic topology based on analyses of all three plant genomes, we do not consider this to be a

484 potential bias (i.e. the AT reflecting the signal of these genes), but instead indicative of our

485 dataset converging on the signal of the true relationships. In our analysis, however, longer genes

486 still had largely discordant trees.

487 The superior performance of (coding) nucleotide data compared to amino acid data

488 possibly stems from the relatively greater information content of nucleotides (i.e., longer

489 alignments). Assuming there is not a significant amount of missing/indel data (with the exception

490 of $y c f 1$ and 2), longer alignments should result in better-informed models of evolution, all else

491 being equal, aided by both parsimony-informative and -uninformative characters (Yang, 1998).

492 However, inherent differences in amino acid and nucleotide models might also explain

493 differences in performance. The nucleotide data were analyzed under the GTR model, where

494 substitution rates among bases are individually estimated; in contrast, for the empirical amino

495 acid substitution models investigated here, substitution rates are pre-estimated, as the number of

496 estimated parameters for changes among 20 states is extremely large. Additionally, although the 
497 plastome has been treated as a single molecule for designing amino acid models of evolution

498 (Adachi et al., 2000), a wide variety of amino acid models (some of which were designed for

499 viruses, such as flu or HIV) were inferred to be the best for different plastid genes (Table S12).

500 This might be the result of the different methods implemented in RAxML vs. IQ-TREE for

501 model testing, the different available models (the best-fitting model for some genes in the

502 RAxML analysis was the unpublished matrix DUMMY2), or the lack of sufficient information

503 (because of gene length) to inform the model. However, the most important point is that, based

504 on the amount of information present in each gene, the chloroplast is inferred to evolve under

505 significantly different models of evolution. Given the highly pectinate structure of the AT,

506 phylogenetic inference in this case should rely heavily on the model for the likelihood

507 calculations. While in some cases (e.g., the shortest plastid genes) limited amounts genetic

508 information might be inherently insufficient, in others, improvements in amino acid modeling

509 might lead to great improvements in phylogenetic inference; such has been suggested for animal

510 mitochondrial data (Richards et al., 2018). At least insofar as the best-fitting model adequately

511 models the dynamics of sequence evolution, conflict would be expected to decrease relative to a

512 sub-optimal model if systematic error (through inadequate modeling) was the main driver of our

513 results. However, we found that our results were recapitulated when analyzed using only the

514 WAG model, suggesting that this was not the case. These analyses are not exhaustive, because

515 they can only testify to the impact of systematic error resulting from the variation among the

516 model set considered here and do not consider the adequacy of these models. Even though two

517 genes analyzed under two different models produce the same tree, there could still be common

518 assumptions underlying both models that are violated by the dataset, leading to systematic error.

519 Additionally, two genes may share the best-fitting model and produce the same tree, but that

520 model may be misspecified, leading to error. Although not the focus of our study, future

521 examinations of chloroplast phylogenomics could benefit from tests of model adequacy (Brown,

522 2014; Duchêne et al., 2018; Chen et al., 2019).

523 We expect that at deeper time scales, nucleotides (of coding regions) may begin to

524 experience saturation and thus information loss due to increased noise, at which point amino

525 acids (with 20 states) would begin to outperform nucleotides. However, the time scale of

526 angiosperm evolution does not appear great enough to result in nucleotide saturation (at least for

527 the genes sampled here, given that no genes appeared to exhibit significant levels of saturation; 
528 Fig. S5), indicating that nucleotides outperform amino acids for phylogenetic analysis of

529 plastomes across angiosperms. Future work, with a broader plastome sampling across green

530 plants, will be necessary to determine the evolutionary scale at which amino acids become more

531 informative than nucleotides for phylogenetic inference.

532 Taxon sampling may also affect the accuracy of phylogenetic inference, and it is possible

533 that some of the incongruence observed here is due to the influence of missing taxa (Zwickl and

534 Hillis, 2002; Heath, Hedtke and Hillis, 2008). There is debate about the relative impact of more

535 sequences versus more taxa (Rosenberg and Kumar, 2001; Zwickl and Hillis, 2002; Rokas and

536 Carroll, 2005). However, our dataset sampled most major angiosperm lineages, and the total

537 analysis was highly concordant with the AT and the GT. Furthermore, patterns of conflict in this

538 dataset reflect those detected in more targeted sampling efforts (Foster et al., 2018). Taken

539 together, this suggests that taxon sampling did not play a large role in our results.

540

541 Analyses of well-supported conflict-The use of a reference topology (AT) afforded us the

542 ability to examine how individual genes within the plastomes agree/conflict with our current,

543 generally accepted hypothesis of angiosperm phylogeny. However, several contentious areas of

544 angiosperm phylogeny remain unresolved, with different topologies recovered across different

545 analyses (e.g., the AT, GT, and PT show small differences in several parts of the tree). Thus, we

546 used edge-based analyses to compare these alternative topologies.

547 The placement of Buxus (Buxales) is a salient example, with different analyses resolving

548 different placements. Our edge-based analyses found Buxus sister to

549 Trochodendron/Trochdendrales (i.e., the GT topology) to have the highest likelihood score

550 (Table 1), and this placement was recovered in four plastid genes ( $r b c L$, petL, rps2, rps 14) with

551 strong support. However, five plastid genes recovered, with strong support, Buxus and

552 Trochodendron successively sister to the core eudicots (i.e., the AT topology): $n d h D$, rps 4 ,

$553 m a t K, p s b B$, and $r p o C 2$. Relationships among core lamiid orders are another intriguing example

554 of gene tree conflict. Core lamiid relationships have been notoriously problematic (Refulio-

555 Rodriguez and Olmstead, 2014; Stull et al., 2015), and previous phylogenetic studies have

556 recovered every possible combination of the constituent orders (Boraginales, Gentianales,

557 Lamiales, Solanales). While our sampling does not include Boraginaceae, we tested alternative

558 relationships among the three remaining orders. The relationship 
559 (Lamiales(Gentianales,Solanales)), which is present in the GT, received the highest likelihood 560 support and is found in 15 gene trees (Tables 1 and 2). However, the alternatives were each 561 recovered with strong support by two plastid genes: psaA and atpH (Gentianales sister to the 562 rest), and $p s b B$ and petA (Solanales sister to the rest). Asteraceae also exhibited several examples 563 of strongly supported conflict (Tables 1 and 2), but sampling differences across the trees

564

565

566

567

568

569

570

571

572

573

574

575

576

577

578

579

580

581

582

583

584

585

586

587

588

589 examined make comparisons more difficult.

It is difficult to determine the causes of the observed instances of strongly supported conflict, which can be found at most nodes in the phylogeny (Fig. 1, Tables S11-12). Based on the present analyses, we cannot conclude whether this conflict is biological (e.g., genes with different evolutionary histories in a single genome) or a consequence of systematic, stochastic, or other analytical errors (e.g., modeling error, lack of information or misalignment). We have endeavored to control for these factors. For example, it is possible that alignment errors could be responsible for instances of strongly supported conflict (Richards et al., 2018). However, amongst genes with strongly supported conflict, no obvious misalignment was present, and recapitulation of the results when testing multiple alignment methods and a cleaning procedure suggests misalignment, if present, does not explain the patterns observed here. Unlike in that study (Richards et al. 2018) supported by a lack of evidence for recombination in animal mitochondria (White et a. 2008), there are reasons to believe that biological gene tree conflict could occur in the chloroplast genome (Sancho et al., 2018), but we stress that we make no attempt to fully characterize this. In parallel to our work, Gonçalves et al. (2019) have analyzed the prevalence of conflict across the plastome and have advocated for the use of both concatenation and multispecies coalescent methods (MSC) for inferring full plastome relationships. Our own analysis of the data with the summary coalescent method ASTRAL (Zhang et al., 2018), demonstrates that the different methods result in different relationships (Fig. 1). Specifically, we found much of the conflict co-occurs with conflict from the full plastome supermatrices, with the exception of the generic level resolutions of Diplostephium. We note that a core assumption of the MSC is the lack of linkage amongst loci, likely violated by the chloroplast in all but a small number of cases (Edwards et al., 2016; Gonçalves et al., 2019). See Gonçalves et al. (2019) for an overview of coalescent methods applied to plastome data. With our data, no analyses we performed fully explained the level of conflict observed and we suggest that the possibility of biological conflict deserves further exploration, especially given the recent 
590 documented instances of inter-plastome recombination (Sullivan et al., 2017; Sancho et al.,

591 2018) and exchange between the chloroplast and other genomes (Martin et al., 1998; Martin

592 2003; Straub et al., 2013).

593

594 Implication for chloroplast phylogenomics - These examples above are not meant to represent 595 an exhaustive examination of conflicting relationships within angiosperms. Instead, they are 596 intended to highlight several instances of strongly supported conflict within the plastome. These 597 results nevertheless have important implications for plastid phylogenomics, suggesting that 598 concatenated analyses should be performed with caution given the notable presence of gene-tree 599 conflict. The majority of nodes show at least one strongly supported conflicting gene, and many

600 nodes have roughly equivalent numbers of conflicting and concordant genes against the 601 reference tree (Fig. 1). Because of the character of the chloroplast, it has been common for 602 authors to assume that gene tree incongruence occurs due to stochastic error, and thus perform 603 concatenation analyses on the whole chloroplast to increase phylogenetic signal. One of the key 604 assumptions of concatenation methods is that all genes share the same underlying tree. While the 605 signal of stochastic error (e.g., longer genes being more concordant) supports this approach, our 606 detection of supported conflict suggests that this approach may be less valid than previously 607 assumed. Concatenated analyses with extensive underlying conflict can yield problematic results 608 in terms of both topology and branch lengths (Brown and Thomson, 2018; Shen et al., 2017;

609 Walker et al., 2018). Particularly against a backdrop of largely uninformative genes, a few genes 610 exhibiting conflict due to stochastic error, inadequate modeling, or biological sources can drive

611 the analysis toward the wrong topology (Brown and Thomson, 2018; Shen et al., 2017; Walker et

612 al., 2018). We suggest that authors should proceed with caution when assuming that all

613 chloroplast genes share the same tree.

614

615 Utility of individual plastid genes for future studies-Previous studies have laid a strong 616 framework for determining the utility of chloroplast regions at various phylogenetic scales. For 617 example, work by Shaw et al. $(2005,2007,2014)$ highlighted non-coding DNA regions useful 618 for shallow evolutionary studies, while Graham and Olmstead (2000) explored protein-coding 619 genes useful for reconstructing deep relationships in angiosperms. Here, we expand upon 620 previous work by using a novel phylogenomic approach, allowing us examining the concordance 
621 of individual protein-coding plastid genes with all nodes of the accepted angiosperm phylogeny

622 (AT; as well as slight variations thereof, e.g., the GT). We paid special attention to matK and

$623 r b c L$, given their historical significance for plant systematics (e.g., Donoghue et al., 1992; Chase

624 et al., 1993; Hilu et al., 2003). We find that $r b c L$ performs relatively well in recapitulating the

625 AT - however, matK performs considerably better (i.e., it generally has more nodes concordant

626 with the AT; Tables S11-12). This is likely due to a strong positive correlation between

627 alignment length and number of concordant nodes, as noted above (Fig. 2); matK has a longer

628 alignment/gene length than $r b c L$.

629 The gene $y c f l$ has been found to be a useful marker in phylogenetics (e.g., Neubig et al.,

630 2008; Neubig and Abbot, 2010; Thomson et al., 2018) and barcoding (Dong et al. 2015), and

631 here we find that it generally performs above average. The alignment of $y c f l$ is abnormally long

632 and contains a large number of gaps, and this is likely due to its position spanning the boundary

633 of the IR and the SSC, an area known to fluctuate greatly in size. This variability likely

634 contributes to the value of $y c f l$ as a marker for 'species-level' phylogenetics and barcoding.

635 However, the performance of $y c f 1$ does not scale with its alignment length. In terms of

636 concordance, we find that matK performs roughly equally as well if not slightly better than $y c f 1$

637 (Tables S11-12). This might in part be a consequence of $y c f l$ being missing/lacking annotation

638 from some species, preventing us from analyzing its concordance/conflict with certain nodes.

639 Nevertheless, our results add to the body of evidence supporting $y c f 1$ as a generally useful plastid

640 region. However, we found $r p o C 2$ to outperform all other plastid regions in every case (Tables

$641 \mathrm{~S} 11-12)$, and its alignment length $(4,660 \mathrm{bp})$ is $\sim 1 / 5^{\text {th }}$ the length of $y c f 1$, easing the computational

642 burden of using alignment tools such as FSA and BAli-Phy (which would struggle with a region

643 as long as $y c f 1$ ), as well as divergence dating and tree-building programs such as BEAST

644 (Redelings and Suchard 2006; Drummond and Rambaut 2007).

645 In our analyses, when BS support is not considered, rpoC2 performed at least as well if

646 not better than using the concatenation of all chloroplast genes (Fig. 3; Tables S11-12). When

647 support is considered (Fig. 3; Tables S11-12), rpoC2 still remains the best-performing gene, but

648 it performs slightly worse than the concatenation of all chloroplast genes (in terms of number of

649 supported nodes concordant with the AT). The utility of rpoC2 likely stems from its notable

650 length, resulting in a wealth of useful phylogenetic information. Biologically, even long genes

651 may be functionally constrained such that not all sites are variable, such as $r b c L$, which 
652 performed relatively poorly here (Kellogg and Juliano, 1997). However, were this to be the case 653 for $r p o C 2$, its longer length relative to $r b c L$ should be expected to counter this effect. In light of 654 our results, $r p o C 2$ should be a highly attractive coding region for future studies, as it generally 655 recapitulates the plastome phylogeny while allowing more proper branch length inferences 656 (given that conflicting signal among multiple genes can result in problematic branch length 657 estimates: Mendes and Hahn, 2016). Recent work (Salichos and Rokas, 2013; Smith et al., 658 2018b; Steenwyk et al., 2018) indicates that filtering for a smaller set of highly informative 659 genes might yield more accurate results in various phylogenetic applications (e.g., topology 660 estimation, divergence dating). Given this, $r p o C 2$ might be particularly useful for comparative 661 analyses requiring accurate branch length estimates (i.e., the majority of comparative analyses).

662 Use of rpoC2 alone (instead of the entire plastome) would also allow for more complex, 663 computationally expensive models to be implemented. Furthermore, focused sequencing of 664 rpoC2 would increase compatibility of datasets from different studies, facilitating subsequent 665 comprehensive, synthetic analyses. Although the performance rpoC2 may be dataset dependent, 666 our results support its utility at multiple levels, in terms of both time scales and sampling. It is important to note, however, that we did not include non-coding regions in our study, which would likely outperform coding regions at shallow phylogenetic levels. As previously noted, however, it is generally too difficult to use non-coding regions across broad phylogenetic scales (e.g., angiosperms) because of complications related to homology assessment and alignment.

672 Genomic patterns of concordance/conflict-Several previous studies (e.g., Jian et al., 2008;

673 Moore et al., 2011) have highlighted the Inverted Repeat (IR) as a valuable plastid region for 674 deep-level phylogenetic analyses, attributing its utility to its relatively slow rate of evolution, 675 resulting in less homoplasy and minimal saturation. Our results contradict this notion, suggesting 676 that the coding sequences of the IR alone perform poorly compared to the LSC and SSC coding 677 regions for reconstructing angiosperm phylogeny (Fig. S1 and Table S13). It is important to note 678 that there are important differences between our study and earlier studies on the IR (e.g., Jian et 679 al., 2008; Moore et al., 2011). For one, we did not include the ribosomal RNA genes, which are 680 highly conserved; thus if the conserved nature of the IR (or at least portions of it) is the basis of 681 its utility, then this might explain the poor performance in the current study. Our saturation 682 analyses, however, (described above) did not reveal any genes to have significant saturation 
683 issues at the scale of angiosperm evolution. This calls into question the idea that the conserved 684 genes of the IR would make it superior for reconstruction of angiosperm phylogeny. Instead, it is 685 possible that the non-coding regions of the IR (which we did not include here) are highly 686 informative for angiosperm phylogeny. While the non-coding regions of the LSC and SSC 687 regions have been extensively examined for use as phylogenetic markers (Shaw et al., 2005, 688 2007, 2014), the non-coding regions of the IR have been underexplored. Among the IR genes 689 examined here, $y c f 2$ (which is exceptionally long) showed the greatest levels of concordance 690 (Fig. S1 and Tables S11-12), underscoring the idea that longer genes are generally more useful 691 for phylogeny reconstruction.

692 Another important difference between our study and Moore et al. (2011) is that we only 693 partitioned our data by gene region, while Moore et al. (2011) explored various partitioning 694 strategies (including codon positions and different combinations of genes). It is clear that 695 sequences within the plastomes follow various different models of molecular evolution (as 696 shown above in the results section Patterns of conflicting chloroplast signal). Exploration of 697 more complicated partitioning schemes-which is a time-consuming process (Lanfear et al., 698 2012; Kainer and Lanfear 2015), and beyond the scope of this study - might generally improve 699 plastome-inferred phylogenies.

701 Conclusions - We find notable levels of gene-tree conflict within the plastome, at all levels of 702 angiosperm phylogeny, highlighting the necessity of future research into the causes of plastome 703 conflict: do some genes share different evolutionary histories, or is systematic error (e.g., poor 704 modeling) the source of the observed conflict? With rare exceptions, most genes are largely 705 uninformative and, when analyzed as a whole, are therefore unlikely to mislead researchers. 706 However, our results call into question the appropriateness of concatenating plastid genes for 707 phylogeny reconstruction without some level of scrutiny. Even if the concatenated topology is 708 largely congruent with the 'true' species tree of a given group, underlying conflict can mislead 709 branch length estimation (Mendes and Hahn, 2016). Therefore, we recommend that comparative 710 methods and/or dating analyses that rely on branch lengths use filtering procedures similar to

711 those proposed for nuclear data (Salichos and Rokas, 2013; Smith et al., 2018b; Steenwyk et al., 712 2018). 
713 We find alignment length and molecule type (nucleotide vs amino acids) to be the

714 strongest correlate with plastid gene performance-i.e., longer genes and nucleotides (which

715 have greater information content than amino acids) generally show higher levels of concordance

716 with our accepted hypothesis of angiosperm phylogeny. This also shows that as a single unit,

$717 m a t K, r b c L$ and the other commonly used marker $n d h F$ are not up to the task alone and if

718 possible should be replaced by longer genes such as $r p o C 2, y c f 1$, and $y c f 2$. Our findings support

719 the notion that longer genes (with greater information content) are generally superior for

720 phylogeny reconstruction, in line with theoretical predictions (Yang, 1998) and should be

721 prioritized when developing phylogenetic studies.

722

\section{ACKNOWLEDGEMENTS}

724 We thank S. A. Smith and M. J. Moore for reading an early draft of the manuscript and proving

725 helpful suggestions toward its improvement. We also thank A. Alverson, Liming Cai, two

726 anonymous reviewers and S. Edwards for helpful comments that improved the manuscript. JFW

727 was supported by a Rackham Predoctoral Fellowship. G.W.S. was supported by an NSF

728 Postdoctoral Fellowship (NSF DBI grant 1612032). O.M.V was supported by NSF FESD

7291338694 and DEB 1240869. D.A.L. was supported by NSF DEB grant 1458466.

730

731

\section{LITERATURE CITED}

732

Adachi, J., P. J. Waddell, W. Martin, and M. Hasegawa. 2000. Plastid genome phylogeny and a

733 model of amino acid substitution for proteins encoded by chloroplast DNA. Journal of

734 Molecular Evolution 50: 348-358.

735

Bendich, A. J. 2004. Circular chloroplast chromosomes: the grand illusion. The Plant Cell 16: $1661-1666$.

737

Birky, C. W., Jr. 1995. Uniparental inheritance of mitochondrial and chloroplast genes:

738 mechanisms and evolution. Proceedings of the National Academy of Sciences 92: 1133111338.

740

Boyce, Kieran, Fabian Sievers, and Desmond G. Higgins. "Instability in progressive multiple sequence alignment algorithms." Algorithms for molecular biology 10, no. 1 (2015): 26.

Bradley, R. K., A. Roberts, M. Smoot, S. Juvekar, J. Do, C. Dewey, I. Holmes, and L. Pachter. 2009. Fast statistical alignment. PLoS Computational Biology 5: e1000392. 
744 Brown, J. M. 2014. Detection of implausible phylogenetic inferences using posterior predictive

745

746

747

748

749

750

751

752

753

754

755

756

757

758

759

760

761

762

763

764

765

766

767

768

769

770

771

772 assessment of model fit. Systematic Biology 63. 334-348.

Brown, J. M., and R. C. Thomson. 2017. Bayes factors unmask highly variable information content, bias, and extreme influence in phylogenomic analyses. Systematic Biology 66: 517530.

Brown, J. W., J. F. Walker, and S. A. Smith. 2017. Phyx: phylogenetic tools for unix. Bioinformatics 33: 1886-1888.

Burleigh, J. G., and S. Mathews. 2007a. Assessing among-locus variation in the inference of seed plant phylogeny. International Journal of Plant Sciences 168: 111-124.

Burleigh, J. G., and S. Mathews. 2007b. Assessing systematic error in the inference of seed plant phylogeny. International Journal of Plant Sciences 168: 125-135.

Carbonell-Caballero, J., R. Alonso, V. Ibañez, J. Terol, M. Talon, and J. Dopazo. 2015. A

Phylogenetic Analysis of 34 Chloroplast Genomes Elucidates the Relationships between Wild and Domestic Species within the Genus Citrus. Molecular Biology and Evolution 32: 20152035.

Chase MW, Soltis DE, Olmstead RG, Morgan D, Les DH, Mishler BD, Duvall MR, Price RA, Hills HG, Qiu YL, Kron KA. Phylogenetics of seed plants: an analysis of nucleotide sequences from the plastid gene rbcL. Annals of the Missouri Botanical Garden. 1993 Jan 1:528-80.Chatzou, M., Floden, E. W., Di Tommaso, P., Gascuel, O., \& Notredame, C. (2018). Generalized bootstrap supports for phylogenetic analyses of protein sequences incorporating alignment uncertainty. Systematic Biology, syx096.

Chen, W., Kenney, T., Bielawski, J., Gu, H. 2019. Testing adequacy for DNA substitution models. BMC Bioinformatics 20. 349.

Corriveau, J. L., and A. W. Coleman. 1988. Rapid screening method to detect potential biparental inheritance of plastid DNA and results for over 200 angiosperms. American Journal of Botany 75: 1443-1458.

Cronn, R., A. Liston, M. Parks, D. S. Gernandt, R. Shen, and T. Mockler. 2008. Multiplex sequencing of plant chloroplast genomes using Solexa sequencing-by-synthesis technology. Nucleic acids research 36: e122-e122. 
773 Cronn, R., B. J. Knaus, A. Liston, P. J. Maughan, M. Parks, J. V. Syring, and J. Udall. 2012.

774

775

776

777

778

779

780

781

782

783

784

785

786

787

788

789

790

791

792

793

794

795

796

797

798

799

800

801

802

Targeted enrichment strategies for next-generation plant biology. American Journal of Botany 99: 291-311.

Csardi, G., Nepusz, T. 2006. The igraph software package for complex network research. Interjournal Complex Systems. 1695.

Davis, Jerrold I., Joel R. Mcneal, Craig F. Barrett, Mark W. Chase, James I. Cohen, Melvin R. Duvall, Thomas J. Givnish, Sean W. Graham, Gitte Petersen, J. Chris Pires, Ole Seberg, Dennis W. Stevenson and Jim Leebens-Mack. "Contrasting Patterns of Support among Plastid Genes and Genomes for Major Clades of the Monocotyledons." Chapter. In Early Events in Monocot Evolution, edited by Paul Wilkin and Simon J. Mayo. Systematics Association Special Volume Series. Cambridge: Cambridge University Press, 2013.

Dong, W., C. Xu, C. Li, J. Sun, Y. Zuo, S. Shi, T. Cheng, J. Guo, and S. Zhou. 2015. ycfl, the most promising plastid DNA barcode of land plants. Scientific Reports 5: 8348.

Donoghue, M. J., R. G. Olmstead, J. F. Smith, and J. D. Palmer. 1992. Phylogenetic relationships of Dipsacales based on rbcL sequences. Annals of the Missouri Botanical Garden 79: 333345.

Doyle, J. J. 1992. Gene trees and species trees: molecular systematics as one-character taxonomy. Systematic Botany 17: 144-163.

Drummond, A. J., and A. Rambaut. 2007. BEAST: Bayesian evolutionary analysis by sampling trees. BMC Evolutionary Biology 7: 214.

Duchêne, D. A., Duchêne, S., Ho, S. Y. W. 2018. Differences in performance among test statistics for assessing phylogenomic model adequacy. Genome Biology and Evolution 10. 1375-1388.

Felsenstein, Joseph, and Gary A. Churchill. "A Hidden Markov Model approach to variation among sites in rate of evolution." Molecular biology and evolution 13, no. 1 (1996): 93-104.

Foster, C.S., Henwood, M.J. and Ho, S.Y., 2018. Plastome sequences and exploration of treespace help to resolve the phylogeny of riceflowers (Thymelaeaceae: Pimelea). Molecular phylogenetics and evolution.

Galtier, N., and V. Daubin. 2008. Dealing with incongruence in phylogenomic analyses.

Philosophical Transactions of the Royal Society B: Biological Sciences 363: 4023-4029.

Peer) reviewing PDF | (2019:05:37731:1:1:REVIEW 24 Jul 2019) 
803 Gitzendanner, M. A., P. S. Soltis, G. K. Wong, B. R. Ruhfel, and D. E. Soltis. 2018. Plastid 804 phylogenomic analysis of green plants: a billion years of evolutionary history. American 805 Journal of Botany 105: 291-301.

806 Gonçalves, Deise JP, Beryl B. Simpson, Edgardo M. Ortiz, Gustavo H. Shimizu, and Robert K. 807

808

809 Jansen. "Incongruence between gene trees and species trees and phylogenetic signal variation in plastid genes." Molecular phylogenetics and evolution (2019).

Graham, S. W., and R. G. Olmstead. 2000. Utility of 17 chloroplast genes for inferring the phylogeny of the basal angiosperms. American Journal of Botany 87: 1712-1730.

Fruchterman, T. M. J., Reingold, E. M. 1991. Graph drawing by force-directed placement. Software: Practice and Experience 21. 1129-1164.

Heath, Tracy A., Shannon M. Hedtke, and David M. Hillis. Taxon sampling and the accuracy of phylogenetic analyses. J Syst Evol 46, no. 3 (2008): 239-257.

Hensen, A.K., L. K. Escobar, L. E. Gilbert, and R. K. Jansen. 2007. Paternal, maternal, and bipaternal inheritance of the chloroplast genome in Passiflora (Passifloraceae): implications for phylogenetic studies. American Journal of Botany 94: 42-46.

Hillis, D. M., J. J. Bull. An empirical test of bootstrapping as a method for assessing confidence in phylogenetic analysis. Systematic Biology 42: 182-192.

Hilu, K. W., T. Borsch, K. Müller, D. E. Soltis, P. S. Soltis, V. Savolainen, M. W. Chase, M. P. 825 Jansen, R. K., Z. Cai, L. A. Raubeson, H. Daniell, C. W. dePamphilis, J. Leebens-Mack, K. F. 826 Müller, M. Guisinger-Bellian, R. C. Haberle, and A. K. Hansen. 2007. Analysis of 81 genes 827 from 64 plastid genomes resolves relationships in angiosperms and identifies genome-scale 828 evolutionary patterns. Proceedings of the National Academy of Sciences 104: 19369-19374. 829 Jian, S., P. S. Soltis, M. A. Gitzendanner, M. J. Moore, R. Li, T. A. Hendry, Y. L. Qiu, A. 830 Dhingra, C. D. Bell, and D. E. Soltis. 2008. Resolving an ancient, rapid radiation in 831 Saxifragales. Systematic Biology 57: 38-57.

832 Johnson, L. B., and J. D. Palmer. 1988. Heteroplasmy of chloroplast DNA in Medicago. Plant 833 Molecular Biology 12: 3-11. 
834 Kainer, D., and R. Lanfear. 2015. The effects of partitioning on phylogenetic inference.

835 Molecular Biology and Evolution 32: 1611-1627.

836 Kalyaanamoorthy, S., B. Q. Minh, T. K. F. Wong, A. von Haeseler, and L. S. Jermiin. 2017.

837 ModelFinder: fast model selection for accurate phylogenetic estimates. Nature Methods 14: $838 \quad 587-589$.

839 Katoh, Kazutaka, and Daron M. Standley. "MAFFT multiple sequence alignment software

840 version 7: improvements in performance and usability." Molecular biology and evolution 30, 841 no. 4 (2013): 772-780.

842 Kellogg, Elizabeth A., and Nickolas D. Juliano. "The structure and function of RuBisCO and 843 their implications for systematic studies." American journal of botany 84, no. 3 (1997): 413844428.

845 R. Lanfear, B. Calcott, S. Y. Ho, and S. Guindon. 2012. PartitionFinder: combined selection of 846 partitioning schemes and substitution models for phylogenetic analyses. Molecular Biology 847 and Evolution 29: 1695-1701.

848 Lee, D. J., T. K. Blake, and S. E. Smith. 1988. Biparental inheritance of chloroplast DNA and the 849 existence of heteroplasmic cells in alfalfa. Theoretical and Applied Genetics 76: 545-549.

850 Li, Hong-Tao, Ting-Shuang Yi, Lian-Ming Gao, Peng-Fei Ma, Ting Zhang, Jun-Bo Yang, 851 Matthew A. Gitzendanner, Peter W Fritsch, Jie Cai, Yang Luo, Hong Wang, Michelle van der 852 Bank, Shu-Dong Zhang, Qing-Feng Wang, Jian Wang, Zhi-Rong Zhang, Chao-Nan Fu, Jing 853 Yang, Peter M. Hollingsworth, Mark W. Chase, Douglas E. Soltis, Pamela S. Soltis and DeZhu Li. "Origin of angiosperms and the puzzle of the Jurassic gap." Nature plants 5, no. 5 (2019): 461.

Liu, J., Z. C. Qi, Y. P. Zhao, C. X. Fu CX, and Q. Y. Jenny Xiang. 2012. Complete cpDNA genome sequence of Smilax china and phylogenetic placement of Liliales-Influences of gene partitions and taxon sampling. Molecular Phylogenetics and Evolution 64: 545-562. "Phylogeny of flowering plants by the chloroplast genome sequences: in search of a "lucky gene"." Biochemistry (Moscow) 72, no. 12 (2007): 1324-1330.

Maddison, W. P. 1997. Gene trees in species trees. Systematic Biology 46: 523-536. 
863 Magallón, S., S. Gómez-Acevedo, L. L. Sánchez-Reyes, T. Hernández-Hernández. 2015. A

864 metacalibrated time-tree documents the early rise of flowering plant phylogenetic diversity.

$865 \quad$ New Phytologist 207: 437-453.

866 Martin, W. 2003. Gene transfer from organelles to the nucleus: frequent and in big chunks.

867 Proceedings of the National Academy of Sciences 100: 8612-8614.

868 Martin, W., B. Stoebe, V. Goremykin, S. Hapsmann, M. Hasegawa, and K. V. Kowallik. 1998.

869 Gene transfer to the nucleus and the evolution of chloroplasts. Nature 393: 162-165.

870 McCauley, D. E., A. K. Sundby, M. F. Bailey MF, M E. Welch. 2007. Inheritance of chloroplast

871 DNA is not strictly maternal in Silene vulgaris (Caryophyllaceae): evidence from

872 experimental crosses and natural populations. American Journal of Botany 94: 1333-1337.

873 Mendes, F. K., and M. H. Hahn. 2016. Gene tree discordance causes apparent substitution rate

874 variation. Systematic Biology 65: 711-721.

875 Mogensen, H. L. 1996. The hows and whys of cytoplasmic inheritance in seed plants. American

876 Journal of Botany 83: 383-404

877 Moore, M. J., A. Dhingra, P. S. Soltis, R. Shaw, W. G. Farmerie, K. M. Folta, and D. E. Soltis.

878 2006. Rapid and accurate pyrosequencing of angiosperm plastid genomes. BMC Plant

879 Biology 6: 17.

880 Moore, M. J., C. D. Bell, P. S. Soltis, and D. E. Soltis. 2007. Using plastid genome-scale data to

881 resolve enigmatic relationships among basal angiosperms. Proceedings of the National

$882 \quad$ Academy of Sciences 104: 19363-19368.

883 Moore MJ, Soltis PS, Bell CD, Burleigh JG, Soltis DE. 2010. Phylogenetic analysis of 83 plastid

884 genes further resolves the early diversification of eudicots. Proceedings of the National

885 Academy of Sciences 107: 4623-4628.

886 Moore, M. J., N. Hassan, M. A. Gitzendanner, R. A. Bruenn, M. Croley, A. Vandeventer, J. W.

887 Horn, A. Dhingra, S. F. Brockington, and M. Latvis. 2011. Phylogenetic analysis of the

888 plastid inverted repeat for 244 species: insights into deeper-level angiosperm relationships

889 from a long, slowly evolving sequence region. International Journal of Plant Sciences 172:

$890 \quad 541-558$.

891 Morrison, David A. "Why would phylogeneticists ignore computerized sequence alignment?."

$892 \quad$ Systematic biology 58, no. 1 (2009): 150-158. 
893 Morrison, David A., Matthew J. Morgan, and Scot A. Kelchner. "Molecular homology and 894 multiple-sequence alignment: an analysis of concepts and practice." Australian Systematic 895 Botany 28, no. 1 (2015): 46-62.

896 Neubig, K. M., and J. R. Abbott. 2010. Primer development for the plastid region ycfl in 897 Annonaceae and other magnoliids. American Journal of Botany 97: e52-e55.

898 Neubig, K. M., W. M. Whitten, B. S. Carlsward, M. A. Blanco, L. Endara, N. H. Williams, and 899 M. J. Moore. 2009. Phylogenetic utility of ycf1 in orchids: a plastid gene more variable than 900 matK. Plant Systematics and Evolution 277: 75-84.

901 Nguyen, L.-T., H. A. Schmidt, A. von Haeseler, and B. Q. Minh. 2014. IQ-TREE: a fast and 902 effective stochastic algorithm for estimating maximum-likelihood phylogenies. Molecular 903 Biology and Evolution 32: 268-274.

904 Palmer, J. D. 1983. Chloroplast DNA exists in two orientations. Nature 301: 92-93.

905 Palmer, J. D. 1985. Comparative organization of chloroplast genomes. Annual review of 906 genetics 19: 325-354.

907 Paradis, Emmanuel, and Klaus Schliep. "ape 5.0: an environment for modern phylogenetics and 908 evolutionary analyses in R." Bioinformatics 35, no. 3 (2018): 526-528.

909 Philippe, Hervé, Ulf Sörhannus, Anne Baroin, Roland Perasso, Françoise Gasse, and André

910 Adoutte. "Comparison of molecular and paleontological data in diatoms suggests a major gap

911 in the fossil record." Journal of Evolutionary Biology 7, no. 2 (1994): 247-265.

912 Philippe, H.,H. Brinkmann, D. V. Lavrov, D. T. J. Littlewood, M. Manuel, G. Wörheide, and D.

913 Baurain. 2011. Resolving difficult phylogenetic questions: why more sequences are not

914 enough. PLoS Biology 9: e1000602.

915 R Core Team. 2018. R: A language and environment for statistical computing. R Foundation for 916 Statistical Computing. Vienna, Austria.

917 Redelings, B. 2014. Erasing errors due to alignment ambiguity when estimating positive

918 selection. Molecular Biology and Evolution 31: 1979-1993.

919 Refulio-Rodriguez, N. F., and R. G. Olmstead. 2014. Phylogeny of Lamiidae. American Journal 920 of Botany 101: 287-299.

921 Danny W. Rice, Andrew J. Alverson, Aaron O. Richardson, Gregory J. Young, M. Virginia 922 Sanchez-Puerta, Jérôme Munzinger, Kerrie Barry, Jeffrey L. Boore, Yan Zhang, Claude W.

923 dePamphilis, Eric B. Knox, Jeffrey D. Palmer. "Horizontal transfer of entire genomes via 
924 mitochondrial fusion in the angiosperm Amborella." Science 342, no. 6165 (2013): 14689251473.

926 Richards, E. J., J. M. Brown, A. J. Barley, R. A. Chong, R. C. Thomson. 2018. Variation across

927 mitochondrial gene trees provides evidence for systematic error: How much gene tree

928 variation is biological? Systematic Biology doi:10.1093/sysbio/syy013.

929 Robinson, D. F., Foulds, L. R. 1981. Comparison of phylogenetic trees. Mathematical

930 Biosciences 53: 131-147.

931 Rodríguez-Ezpeleta, N., H. Brinkmann, G. Burger, A. J. Roder, M. W. Gray, H. Philippe, and B.

932 F. Lang. 2007. Toward resolving the eukaryotic tree: the phylogenetic positions of jakobids

933 and cercozoans. Current Biology 17: 1420-1425.

934 Rokas, A., B. L. Williams, N. King, S. B. Carroll. 2003. Genome-scale approaches to resolving 935 incongruence in molecular phylogenies. Nature 425: 798-804.

936 Rokas, Antonis, and Sean B. Carroll. More genes or more taxa? The relative contribution of gene 937 number and taxon number to phylogenetic accuracy. Molecular biology and evolution 22, no. $938 \quad 5(2005): 1337-1344$.

939 Roquet, C., I. Sanmartín, N. Garcia-Jacas, L. Sáez, A. Susanna, N. Wikström, and J. J. Aldasoro.

940 2009. Reconstructing the history of Campanulaceae with a Bayesian approach to molecular

941 dating and dispersal-vicariance analyses. Molecular Phylogenetics and Evolution 52: 575-

942587.

943 Rosenberg, Michael S., and Sudhir Kumar. Incomplete taxon sampling is not a problem for

944 phylogenetic inference. Proceedings of the National Academy of Sciences 98, no. 19 (2001):

945 10751-10756.

946 Ruhfel, B. R., M. A. Gitzendanner, P. S. Soltis, D. E. Soltis, and J. G. Burleigh. 2014. From

947 algae to angiosperms-inferring the phylogeny of green plants (Viridiplantae) from 360 plastid

948 genomes. BMC Evolutionary Biology 14: 23.

949 Salichos, Leonidas, and Antonis Rokas. "Inferring ancient divergences requires genes with

$950 \quad$ strong phylogenetic signals." Nature 497, no. 7449 (2013): 327.

951 Sancho, R., C. P. Cantalapiedra, D. López-Alvarez, S. P. Gordon, J. P. Vogel, P. Catalán, B.

952 Contreras-Moreira. 2018. Comparative plastome genomics and phylogenomics of

953 Brachypodium: flowering time signatures, introgression and recombination in recently

954 diverged ecotypes. New Phytologist 218: 1631-1644. 
955 Shaw, J., E. B. Lickey, J. T. Beck, S. B. Farmer, W. Liu, J. Miller, K. C. Siripun, C. T. Winder, 956 E. E. Schilling, R. L. Small. 2005. The tortoise and the hare II: relative utility of 21 noncoding 957 chloroplast DNA sequences for phylogenetic analysis. American Journal of Botany 92: 142958166.

959 Shaw, J., E. B. Lickey, E. E. Schilling, R. L. Small. 2007. Comparison of whole chloroplast 960 genome sequences to choose noncoding regions for phylogenetic studies in angiosperms: the 961 tortoise and the hare III. American Journal of Botany 94: 275-288.

962 Shaw, J., H. L. Shafer, O. R. Leonard, M. J. Kovach, M. Schorr, and A. B. Morris. 2014.

963 Chloroplast DNA sequence utility for the lowest phylogenetic and phylogeographic

964 inferences in angiosperms: the tortoise and the hare IV. American Journal of Botany 101:

965 1987-2004.

966 Shen, Xing-Xing, Leonidas Salichos, and Antonis Rokas. "A genome-scale investigation of how

967 sequence, function, and tree-based gene properties influence phylogenetic inference."

968 Genome biology and evolution 8, no. 8 (2016): 2565-2580.

969 Shen, X.-X., C. T. Hittinger, and A. Rokas. 2017. Contentious relationships in phylogenomic

970 studies can be driven by a handful of genes. Nature Ecology \& Evolution 1: 126.

971 Shepherd, L. D., B. R. Holland, L. P. Perrie. 2008. Conflict amongst chloroplast DNA sequences

972 obscures the phylogeny of a group of Asplenium ferns. Molecular Phylogenetics and

973 Evolution 48: 176-187.

974 Smith, David Roy. "Mitochondrion-to-plastid DNA transfer: it happens." New Phytologist 202, 975 no. 3 (2014): 736-738.

976 Smith, S. E. 1989. Biparental inheritance of organelles and its implications in crop improvement. 977 Plant Breeding Reviews 6: 361-393.

978 Smith, S. A., M. J. Moore, J. W. Brown, and Y. Yang. 2015. Analysis of phylogenomic datasets 979 reveals conflict, concordance, and gene duplications with examples from animals and plants.

980 BMC Evolutionary Biology 15: 150.

981 Stephen A. Smith, Nathanael Walker-Hale, Joseph F. Walker, Joseph W. Brown. Nested 982 phylogenetic conflicts, combinability, and deep phylogenomics in plants. bioRxiv 371930; 983 doi: https://doi.org/10.1101/371930

984 DOUGLAS E. SOLTIS, PAMELA S. SOLTIS, MARK W. CHASE, MARK E. MORT, DIRK 985 C. ALBACH, MICHAEL ZANIS, VINCENT SAVOLAINEN, WILLIAM H. HAHN, SARA 
986

987

988

989

990

991

992

993

994

995

996

997

998

999

1000

1001

1002

1003

1004

1005

1006

1007

1008

1009

1010

1011

1012

1013

1014

1015

1016

\section{B. HOOT, MICHAEL F. FAY, MICHAEL AXTELL, SUSAN M. SWENSEN, LINDA M.} PRINCE, W. JOHN KRESS, KEVIN C. NIXON, JAMES S. FARRIS, Angiosperm phylogeny inferred from $18 \mathrm{~S}$ rDNA, $r b c L$, and $a t p B$ sequences, Botanical Journal of the Linnean Society, Volume 133, Issue 4, August 2000, Pages 381-46.

Douglas E. Soltis Stephen A. Smith Nico Cellinese Kenneth J. Wurdack David C. Tank Samuel F. Brockington Nancy F. Refulio-Rodriguez Jay B. Walker Michael J. Moore Barbara S. Carlsward Charles D. Bell Maribeth Latvis Sunny Crawley Chelsea Black Diaga Diouf Zhenxiang Xi Catherine A. Rushworth Matthew A. Gitzendanner Kenneth J. Sytsma Yin-Long Qiu Khidir W. Hilu Charles C. Davis Michael J. Sanderson Reed S. Beaman Richard G. Olmstead Walter S. Judd Michael J. Donoghue Pamela S. Soltis. Angiosperm phylogeny: 17 genes, 640 taxa. American journal of botany. 2011 Apr;98(4):704-30.

Stamatakis, A. 2014. RAxML version 8: a tool for phylogenetic analysis and post-analysis of large phylogenies. Bioinformatics 30: 1312-1313.

Steenwyk, Jacob L., Xing-Xing Shen, Abigail L. Lind, Gustavo G. Goldman, and Antonis Rokas. "A robust phylogenomic timetree for biotechnologically and medically important fungi from Aspergillaceae (Eurotiomycetes, Ascomycota)." bioRxiv(2018): 370429.

Stegemann, S., S. Hartmann, S. Ruf, and R. Bock. 2003. High-frequencing gene transfer from the chloroplast genome to the nucleus. Proceedings of the National Academy of Sciences 100: 8828-8833.

Straub, S, C, K, Cronn, R. C., Edwards, C., Fishbein, M, and A. Liston. 2013. Horizontal Transfer of DNA from the Mitochondrial to the Plastid Genome and Its Subsequent Evolution in Milkweeds (Apocynaceae). Genome Biology and Evolution 5: 1872-1885.

Stull, G. W., M. J. Moore, V. S. Mandala, N. A. Douglas, H. R. Kates, X. Qi, S. F. Brockington, P. S. Soltis, D. E. Soltis, and M. A. Gitzendanner. 2013. A targeted enrichment strategy for massively parallel sequencing of angiosperm plastid genomes. Applications in Plant Sciences 1: 1200497.

Stull, G. W., R. Duno de Stefano, D. E. Soltis, P. S. Soltis. 2015. Resolving basal lamiid phylogeny and the circumscription of Icacinaceae with a plastome-scale data set. American Journal of Botany 102: 1794-1813.

Suchard, M. A., and B. D. Redelings. 2006. BAli-Phy: simultaneous Bayesian inference of alignment and phylogeny. Bioinformatics 22: 2047-2048. 
1017 Sullivan, A. R., B. Schiffthaler, S. L. Thompson, N. R. Street, and X. R. Wang. 2017.

1018 Interspecific plastome recombination reflects ancient reticulate evolution in Picea (Pinaceae).

1019 Molecular Biology and Evolution 34: 1689-1701.

1020 Taberlet, P., L. Gielly, G. Pautou, and J. Bouvet. 1991. Universal primers for amplification of 1021 three non-coding regions of chloroplast DNA. Plant molecular biology 17: 1105-1109.

1022 Thomson, A. M., O. M. Vargas, and C. W. Dick. 2018. Complete plastome sequences from

1023 Bertholletia excelsa and 23 related species yield informative markers for Lecythidaceae.

1024 Applications in Plant Sciences 6: e1151.

1025 Uribe-Convers, S., J. R. Duke, M. J. Moore, D. C. Tank. 2014. A long PCR-based approach for 1026 DNA enrichment prior to next-generation sequencing for systematic studies. Applications in $1027 \quad$ Plant Sciences 2: 1300063.

1028 Vargas, O. M., E. M. Ortiz, and B. B. Simpson. 2017. Conflicting phylogenomic signals reveal a 1029 pattern of reticulate evolution in a recent high-Andean diversification (Asteraceae: Astereae:

1030 Diplostephium). New Phytologist 214: 1736-1750.

1031 Walker, J. F., M. J. Zanis, N. C. Emery. 2014. Comparative analysis of complete chloroplast

1032 genome sequence and inversion variation in Lasthenia burkei (Madieae, Asteraceae)."

1033 American Journal of Botany 101: 722-729.

1034 Walker, J. F., R. K. Jansen, M. J. Zanis, N. C. Emery. 2015. Sources of inversion variation in the

1035 small single copy (SSC) region of chloroplast genomes. American Journal of Botany 102 :

1036 1751-1752.

1037 Walker, J. F., Y. Yang, M. J. Moore, J. Mikenas, A. Timoneda, S. F. Brockington, and S. A.

1038 Smith. 2017. Widespread paleopolyploidy, gene tree conflict, and recalcitrant relationships

1039 among the carnivorous Caryophyllales. American Journal of Botany 104: 858-867.

1040 Walker, J.F., Brown, J.W. and Smith, S.A., 2018. Analyzing contentious relationships and outlier 1041 genes in phylogenomics. Systematic biology 67: 916-924.

1042 Whelan, Simon, and Nick Goldman. "A general empirical model of protein evolution derived 1043 from multiple protein families using a maximum-likelihood approach." Molecular biology 1044 and evolution 18, no. 5 (2001): 691-699.

1045 White, Daniel James, Jonci Nikolai Wolff, Melanie Pierson, and Neil John Gemmell. "Revealing 1046 the hidden complexities of mtDNA inheritance." Molecular Ecology 17, no. 23 (2008): 4925$1047 \quad 4942$. 
1048 Norman J. Wickett, Siavash Mirarab, Nam Nguyen, Tandy Warnow, Eric Carpenter, Naim

1049 Matasci, Saravanaraj Ayyampalayam, Michael S. Barker, J. Gordon Burleigh, Matthew A.

1050 Gitzendanner, Brad R. Ruhfel, Eric Wafula, Joshua P. Der, Sean W. Graham, Sarah Mathews,

1051 Michael Melkonian, Douglas E. Soltis, Pamela S. Soltis, Nicholas W. Miles, Carl J. Rothfels,

1052 Lisa Pokorny, A. Jonathan Shaw, Lisa DeGironimo, Dennis W. Stevenson, Barbara Surek,

1053 Juan Carlos Villarreal, Béatrice Roure, Hervé Philippe, Claude W. dePamphilis, Tao Chen,

1054 Michael K. Deyholos, Regina S. Baucom, Toni M. Kutchan, Megan M. Augustin, Jun Wang,

1055 Yong Zhang, Zhijian Tian, Zhixiang Yan, Xiaolei Wu, Xiao Sun, Gane Ka-Shu Wong, James

1056 Leebens-Mack. Phylotranscriptomic analysis of the origin and early diversification of land

1057 plants. Proceedings of the National Academy of Sciences. 2014 Nov 11;111(45):E4859-68.

1058 Wolfe, K. H., W.-H. Li, and P. M. Sharp. 1987. Rates of nucleotide substitution vary greatly

1059 among plant mitochondrial, chloroplast, and nuclear DNAs. Proceedings of the National

$1060 \quad$ Academy of Sciences 84: 9054-9058.

1061 Wolfe A. D., and C. P. Randle. 2004. Recombination, heteroplasmy, haplotype polymorphism,

1062 and paralogy in plastid genes: implications for plant molecular systematics. Systematic

1063 Botany 29:1011-1020.

1064 Yang, Z. 1998. On the best evolutionary rate for phylogenetic analysis. Systematic Biology 47:

$1065 \quad 125-133$.

1066 Zeng, L., Q. Zhang, R. Sun, H. Kong, N. Zhang, H. Ma. 2014. Resolution of deep angiosperm

1067 phylogeny using conserved nuclear genes and estimates of early divergence times. Nature

$1068 \quad$ Communications 5: 4956.

1069 Zhang, Chao, Maryam Rabiee, Erfan Sayyari, and Siavash Mirarab. "ASTRAL-III: polynomial

1070 time species tree reconstruction from partially resolved gene trees." BMC bioinformatics 19 ,

1071 no. 6 (2018): 153.

1072 Zhang, Q., Y. Liu, and Sodmergen. 2003. Examination of the cytoplasmic DNA in male

1073 reproductive cells to determine the potential for cytoplasmic inheritance in 295 angiosperm

$1074 \quad$ species. Plant and Cell Physiology 44: 941-951.

1075 Zwickl, Derrick J., and David M. Hillis. "Increased taxon sampling greatly reduces phylogenetic

1076 error." Systematic Biology 51, no. 4 (2002): 588-598.

1077

1078

PeerJ reviewing PDF | (2019:05:37731:1:1:REVIEW 24 Jul 2019) 


\section{Figure Legends}

1081 Figure 1. Summary of chloroplast conflict against the reference phylogeny of angiosperms.

1082 Green, orange, and purple boxes indicate where the amino acid, nucleotide-inferred, or

1083 Maximum Quartet Support Species Tree plastome trees conflict with the reference phylogeny.

1084 Pie charts depict the amount of gene tree conflict observed in the nucleotide analysis, with the

1085 blue, green, red and gray slices representing, respectively, the proportion of gene trees

1086 concordant, conflicting (supporting a single main alternative topology), conflicting (supporting

1087 various alternative topologies), and uninformative (BS $<70$ or missing taxon) at each node in the

1088 species tree. The dashed lines represent 30 myr time intervals (positioned based on Magallon et

1089 al. 2015 and Vargas et al. 2017) used to bin nodes for examinations of conflict at different levels

1090 of divergence.

1091

1092 Figure 2. Gene tree concordance/conflict at varying time scales. Each diagram represents a

1093 different molecule type and shows the proportion of concordance each gene exhibits at the five

1094 time slices shown in Fig. 1: (1) 150-120 mya, (2) 120-90 mya, (3) 90-60 mya, (4) 60-30 mya,

1095 (5) 30-0 mya and $C$ is the concordance summed over all the time scales. The individual genes

1096 are scaled by length of alignment; however, $y c f 1$ and $y c f 2$ are cut to approximately the length of

1097 rроC2 due to their extremely long alignments. A Results from amino acid data considering only

1098 nodes with bootstrap support $\geq 70 \%$. B Results from nucleotide data considering only nodes with

1099 bootstrap support $\geq 70 \%$. The plots along the bottom show relationships between gene

1100 concordance levels and alignment length and tree length, excluding outlying genes (see

1101 Methods). Each point represents the proportion of concordance considering only nodes with

1102 bootstrap support $\geq 70 \%$. Red lines show the predicted values from logistic regression and

1103 asterisks give the $\mathrm{p}$-value of the relationship from univariate logistic regression, $* * *=\mathrm{p}<0.001$.

1104 C Logistic regression of concordant nodes from amino acid data against alignment length. D

1105 Logistic regression of concordant nodes from amino acid data against tree length. E Logistic

1106 regression of concordant nodes from nucleotide data against alignment length. F Logistic

1107 regression of concordant nodes from nucleotide data against tree length.

1108 
1109 Figure 3. Histograms depicting number of concordant edges each gene tree contains

1110 compared to the reference phylogeny (i.e., the 'accepted tree, AT). Histograms are binned by

1111 number of concordant nodes, bar heights give the number of genes in each bin. Commonly used

1112 markers ( $m a t K, n d h F, r b c L$, and $y c f 1$ ) are labeled on the graph, along with the most concordant

1113 gene (rpoC2) and the number of concordant nodes for the complete chloroplast (CC) compared

1114 to the AT. A Concordance in the nucleotide dataset not considering bootstrap support. B

1115 Concordance in the amino acid dataset not considering bootstrap support. C Concordance in the

1116 nucleotide dataset considering bootstrap support $\geq 70 \%$. D Concordance in the amino acid

1117 dataset considering bootstrap support $\geq 70 \%$. 


\section{Figure 1}

Figure 1. Summary of chloroplast conflict against the reference phylogeny of angiosperms.

Green, orange, and purple boxes indicate where the amino acid, nucleotide-inferred, or Maximum Quartet Support Species Tree plastome trees conflict with the reference phylogeny. Pie charts depict the amount of gene tree conflict observed in the nucleotide analysis, with the blue, green, red and gray slices representing, respectively, the proportion of gene trees concordant, conflicting (supporting a single main alternative topology), conflicting (supporting various alternative topologies), and uninformative (BS $<70$ or missing taxon) at each node in the species tree. The dashed lines represent 30 myr time intervals (positioned based on Magallon et al. 2015 and Vargas et al. 2017) used to bin nodes for examinations of conflict at different levels of divergence. 


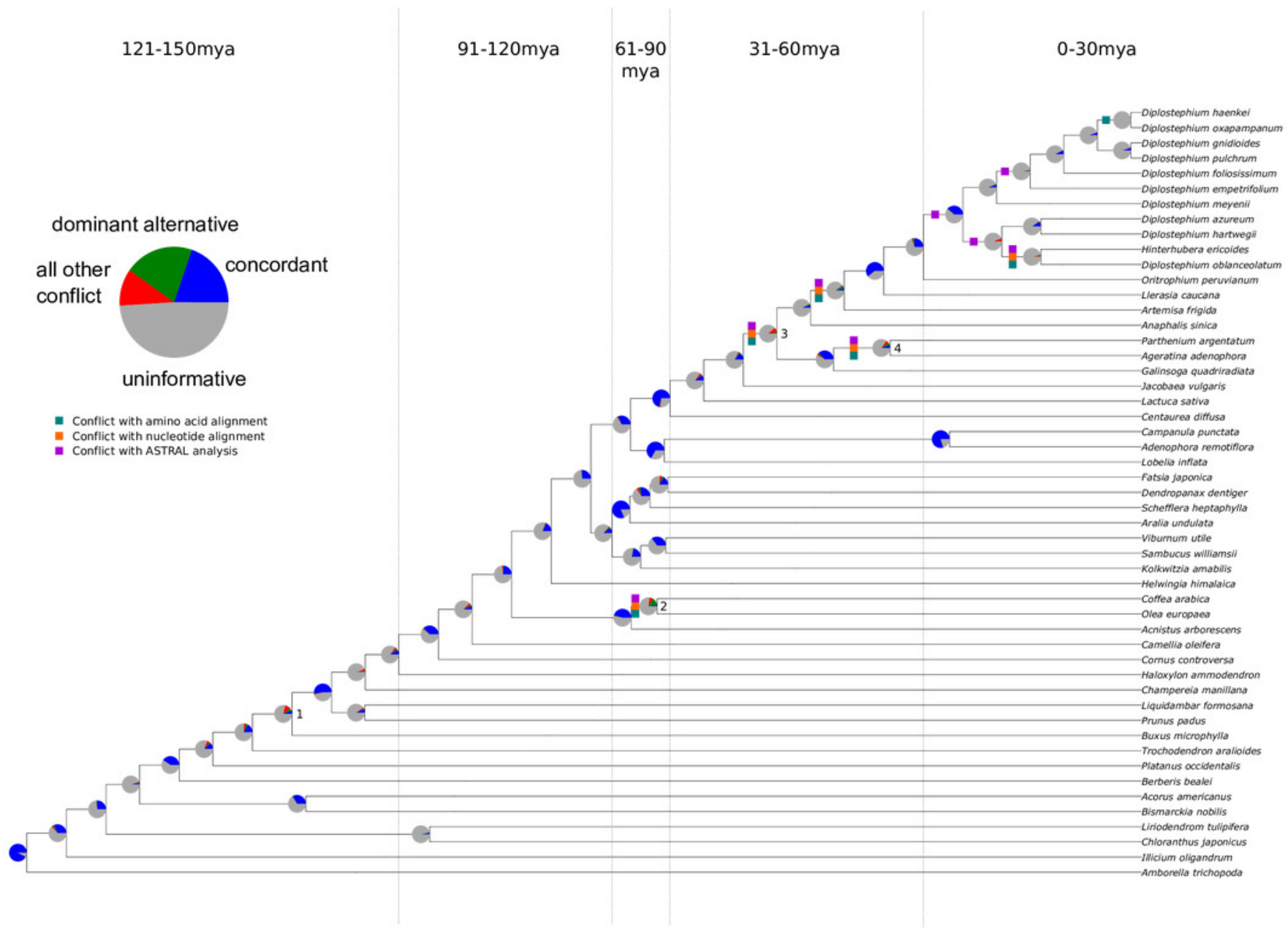




\section{Figure 2}

Figure 2. Gene tree concordance/conflict at varying time scales.

Each diagram represents a different molecule type and shows the proportion of concordance each gene exhibits at the five time slices shown in Fig. 1: (1) 150-120 mya, (2) 120-90 mya, (3) 90-60 mya, (4) 60-30 mya, (5) 30-0 mya and $C$ is the concordance summed over all the time scales. The individual genes are scaled by length of alignment; however, ycf1 and ycf2 are cut to approximately the length of $r p o C 2$ due to their extremely long alignments. A) Results from amino acid data considering only nodes with bootstrap support $\geq 70 \%$. B) Results from nucleotide data considering only nodes with bootstrap support $\geq 70 \%$. The plots along the bottom show relationships between gene concordance levels and alignment length and tree length, excluding outlying genes (see Methods). Each point represents the proportion of concordance considering only nodes with bootstrap support $\geq 70 \%$. Red lines show the predicted values from logistic regression and asterisks give the $p$-value of the relationship from univariate logistic regression, ${ }^{* * *}=p<0.001$. C) Logistic regression of concordant nodes from amino acid data against alignment length. D) Logistic regression of concordant nodes from amino acid data against tree length. E) Logistic regression of concordant nodes from nucleotide data against alignment length. F) Logistic regression of concordant nodes from nucleotide data against tree length. 

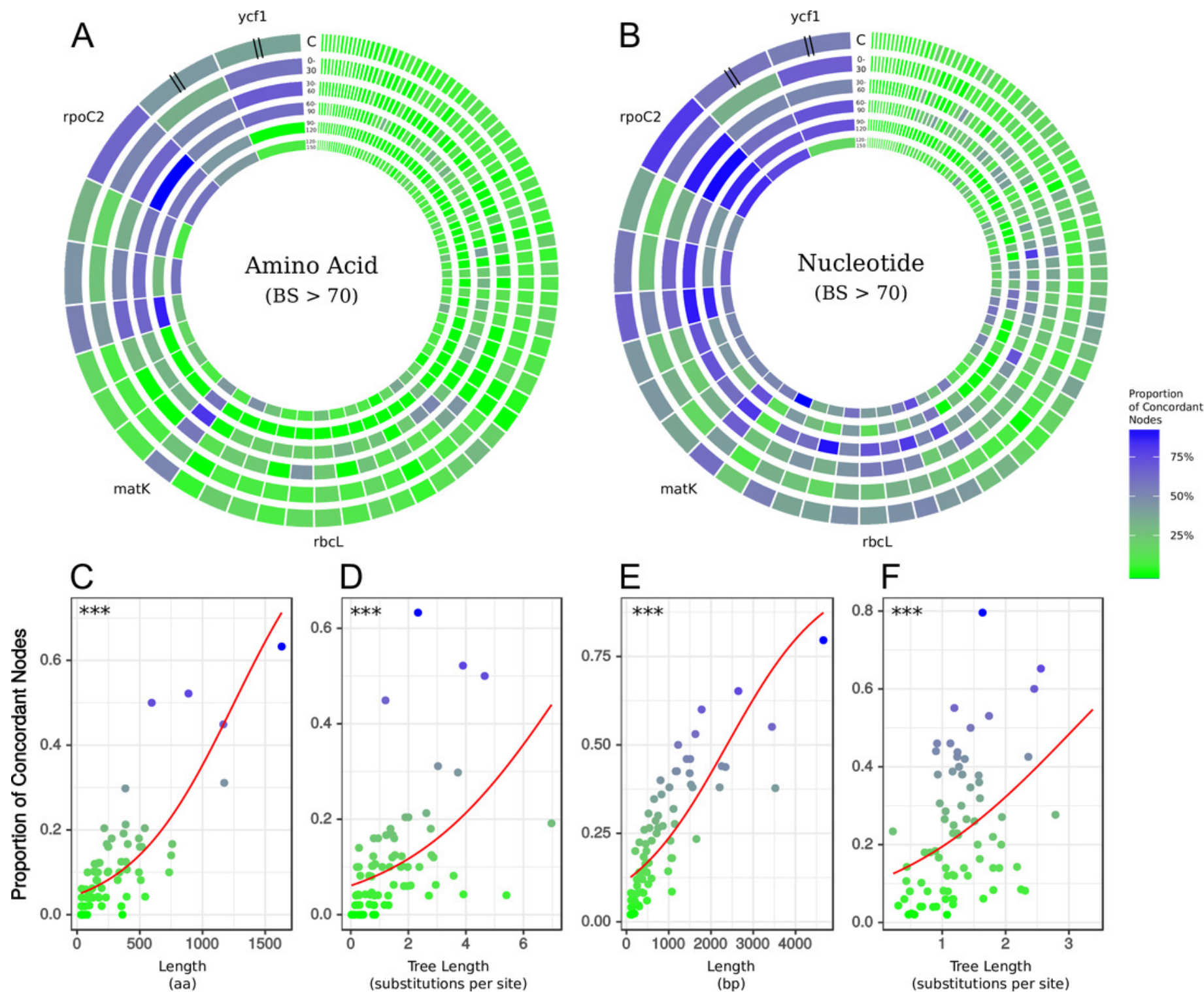

(substitutions per site)

(bp)

(substitutions per site) 


\section{Figure 3}

Figure 3. Histograms depicting number of concordant edges each gene tree contains compared to the reference phylogeny (i.e., the 'accepted tree, AT).

Histograms are binned by number of concordant nodes, bar heights give the number of genes in each bin. Commonly used markers (matK, $n d h F, r b c L$, and $y c f 1$ ) are labeled on the graph, along with the most concordant gene (rpoc2) and the number of concordant nodes for the complete chloroplast (CC) compared to the AT. A) Concordance in the nucleotide dataset not considering bootstrap support. B) Concordance in the amino acid dataset not considering bootstrap support. C) Concordance in the nucleotide dataset considering bootstrap support $\geq$ $70 \%$. D) Concordance in the amino acid dataset considering bootstrap support $\geq 70 \%$.

A

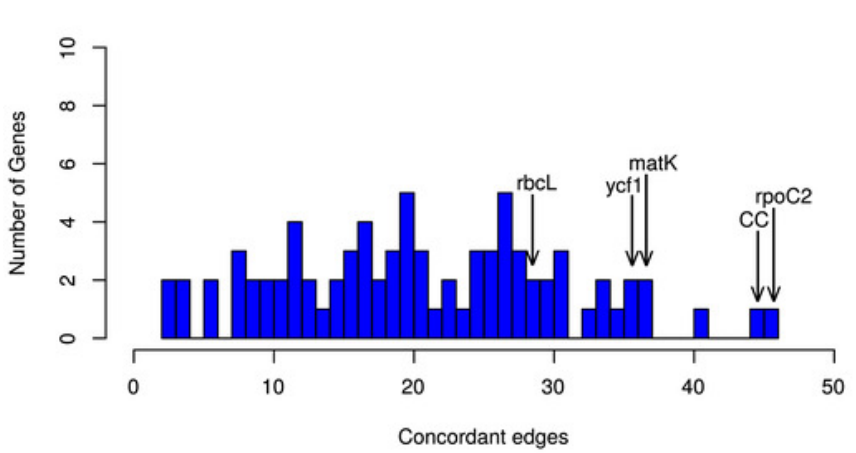

C

Nucleotide

(BS $\geq 70$ )

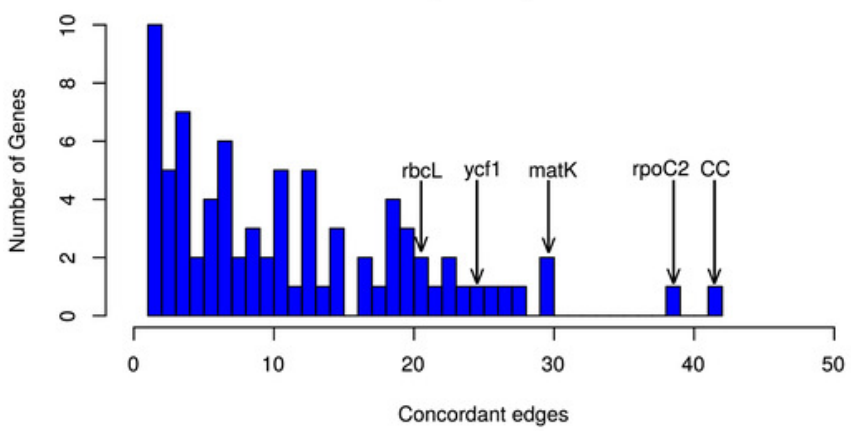

B

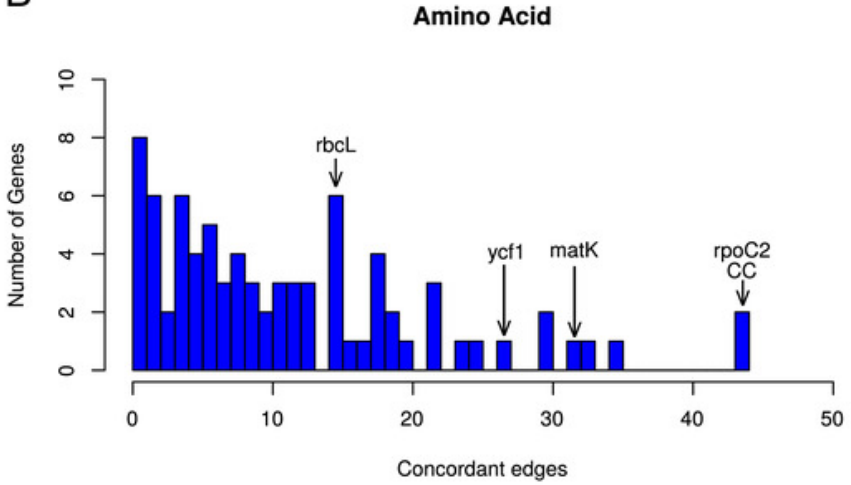

D

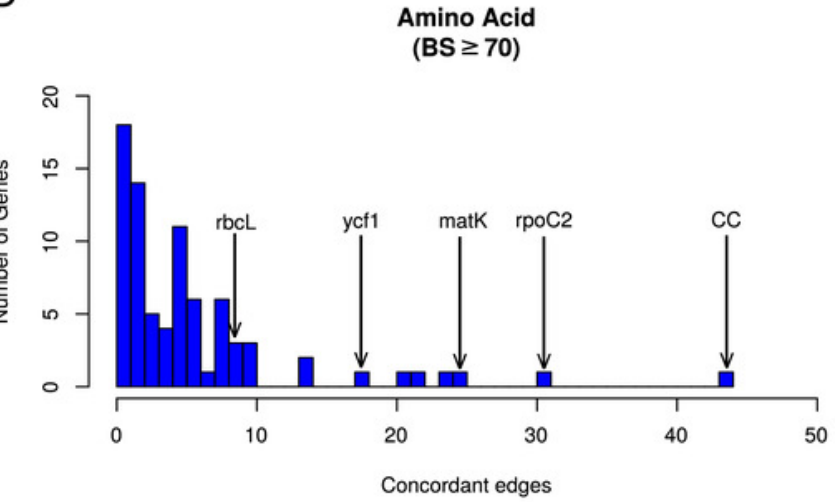




\section{Table $\mathbf{1}$ (on next page)}

Table 1. Log-Likelihood scores from the edge-based analysis.

Log-Likelihood scores rounded to the nearest whole number for alternative resolutions of contentious nodes based on edge-based analyses using a modification of the Maximum Gene Wise Edge (MGWE) method (Walker et al. 2018). The best-supported relationship (highest log-likelihood score) for each case is presented in bold. 
1 Table 1. Log-Likelihood scores rounded to the nearest whole number for alternative resolutions

2 of contentious nodes based on edge-based analyses using a modification of the Maxmimum

3 Gene Wise Edge (MGWE) method (Walker et al. 2018). The best-supported relationship (highest

4 log-likelihood score) for each case is presented in bold.

\begin{tabular}{|l|l|l|l|l|}
\hline $\begin{array}{l}\text { Contentious } \\
\text { relationships }\end{array}$ & GT & AT & CC & $\begin{array}{l}\text { Alternative } \\
\text { topology }(>3 \\
\text { genes supporting) }\end{array}$ \\
\hline $\begin{array}{l}\text { Buxus placement } \\
\text { (node 10) }\end{array}$ & $\mathbf{- 6 1 8 2 4 2}$ & -618371 & -618371 & $\mathbf{- 6 1 8 2 4 2}$ \\
\hline $\begin{array}{l}\text { Lamiid relationships } \\
\text { (node 19) }\end{array}$ & $\mathbf{- 6 1 8 2 0 3}$ & -618235 & $\mathbf{- 6 1 8 2 0 3}$ & $\mathbf{- 6 1 8 2 0 3}$ \\
\hline $\begin{array}{l}\text { Jacobaea } \text { and } \\
\text { Artemisia } \text { placements } \\
\text { (node 34) }\end{array}$ & -618535 & $\mathbf{- 6 1 8 5 0 6}$ & $\mathrm{N} / \mathrm{A} \mathrm{(BS}<70)$ & N/A \\
\hline $\begin{array}{l}\text { Galinsoga } \text { placement } \\
\text { (node 36) }\end{array}$ & $\mathrm{NA}$ & -618308 & $\mathbf{- 6 1 8 1 7 4}$ & -618319 \\
\hline
\end{tabular}

5

6 


\section{Table 2 (on next page)}

Table 2. Contentious relationships tested using edge-based analyses.

Individual genes supporting (with $\geq 70$ BS) the alternative topologies examined for each contentious relationship. 
1 Table 2. Individual genes supporting (with $\geq 70 \mathrm{BS}$ ) the alternative topologies examined for each

2 contentious relationship.

\begin{tabular}{|c|c|c|c|c|}
\hline Contentious relationships & GT & AT & $\mathrm{CC}$ & $\begin{array}{l}\text { Alternative topology } \\
\text { (>3 genes } \\
\text { supporting) }\end{array}$ \\
\hline $\begin{array}{l}\text { Buxus placement } \\
\text { (node } 10 \text { ) }\end{array}$ & $\begin{array}{l}\text { rbcL, petL, rps2, } \\
\text { rps } 14\end{array}$ & $\begin{array}{l}\text { ndhD, rps4, } \\
\text { matK, psbB, } \\
\text { rpoC2 }\end{array}$ & $\begin{array}{l}\text { ndhD, rps4, } \\
\text { matK, psbB, } \\
\text { rpoC2 }\end{array}$ & $\begin{array}{l}r b c L, \text { petL, rps2, } \\
r p s 14\end{array}$ \\
\hline $\begin{array}{l}\text { Lamiid relationships } \\
\text { (node } 19 \text { ) }\end{array}$ & $\begin{array}{l}\text { ndhK, rbcL, } \\
\text { rps } 11, \text { rps } 7, y c f 4, \\
\text { rpoC2, rps } 4, \\
\text { ndhA, ndhJ, ccs } A, \\
\text { rps2, petB, matK, } \\
\text { ndhI, atpB }\end{array}$ & psbB, petA & $\begin{array}{l}n d h K, \operatorname{rbcL}, \\
\operatorname{rps} 11, \operatorname{rps} 7, \\
y c f 4, \operatorname{rpo} C 2, \\
r p s 4, \text { ndhA, } \\
n d h J, \operatorname{ccs} A, \\
\operatorname{rps} 2, \operatorname{pet} B, \\
\operatorname{matK}, \text { ndhI, } \\
\text { atpB }\end{array}$ & $\begin{array}{l}n d h K, \text { rbcL, rps } 11, \\
\text { rps } 7, y c f 4, \text { rpoC } 2, \\
r p s 4, \text { ndhA, ndhJ, } \\
\operatorname{ccs} A, \text { rps } 2, \text { petB, } \\
\text { matK, ndhI, atpB }\end{array}$ \\
\hline $\begin{array}{l}\text { Jacobaea and Artemisia } \\
\text { placements (node } 34 \text { ) }\end{array}$ & rpoC2 & None & N/A & N/A \\
\hline
\end{tabular}

